OPEN ACCESS

Edited by:

Xiang Xue,

University of New Mexico,

United States

Reviewed by:

Fei Teng,

Cornell University, United States

Yuji Naito,

Kyoto Prefectural University of

Medicine, Japan

*Correspondence:

Suhua QI

suhuaqi@zhmu.edu.cn

Bing Gu

binggu2015@xzhmu.edu.cn

tThese authors share first authorship

Specialty section

This article was submitted to

Gastroenterology,

a section of the journal

Frontiers in Medicine

Received: 17 July 2020 Accepted: 17 November 2020 Published: 10 December 2020

Citation:

Yang $\mathrm{H}$, Cai R, Kong Z, Chen Y,

Cheng C, Qi S and Gu B (2020)

Teasaponin Ameliorates Murine Colitis by Regulating Gut Microbiota and Suppressing the Immune System Response. Front. Med. 7:584369. doi: 10.3389/fmed.2020.584369

\section{Teasaponin Ameliorates Murine Colitis by Regulating Gut Microbiota and Suppressing the Immune System Response}

\author{
Huan Yang ${ }^{1 \dagger}$, Rui Cai ${ }^{1 \dagger}$, Ziyan Kong ${ }^{1 \dagger}$, Ying Chen ${ }^{1}$, Chen Cheng ${ }^{1}$, Suhua $Q i^{1 *}$ and \\ Bing $\mathrm{G} u^{1,2 *}$
}

${ }^{1}$ Xuzhou Key Laboratory of Laboratory Diagnostics, Medical Technology School of Xuzhou Medical University, Xuzhou, China, ${ }^{2}$ Department of Laboratory Medicine, Affiliated Hospital of Xuzhou Medical University, Xuzhou, China

Background: Dietary intervention is an exciting topic in current research of inflammatory bowel disease (IBD). The effect of teasaponin (TS) on IBD has not been fully elucidated. Here, we aim to investigate the intestinal anti-inflammatory activity of TS in a dextran sodium sulfate (DSS)-induced colitis mouse model and identify potential mechanisms.

Methods: We applied TS to mice with DSS-induced colitis and then monitored the body weight, disease activity index (DAl) daily. When sacrificed, the intestinal permeability was measured. The analysis of mucin and tight junction proteins was conducted. We detected the inflammatory cytokines, the immune cells and related inflammatory signaling pathways. In addition, the gut microbiota were analyzed by $16 \mathrm{~S}$ rRNA sequencing and we also performed fecal microbiota transplantation (FMT).

Results: It showed that TS ameliorated the colonic damage by lowering the DAI, prolonging the colon length, reducing inflammatory cytokines and improving the mucus barrier. Parallel to down-regulation of the inflammatory cytokines, the fecal lipocalin 2, p-P65, p-STAT3, and neutrophil accumulation were also decreased in TS-treated mice. Microbiota characterization showed that Campylobacteria, Proteobacteria, Helicobacter, and Enterobacteriaceae were the key bacteria associated with IBD. In addition, TS could reverse the Firmicutes/Bacteroidetes (F/B) ratio and increase the beneficial bacteria, including Akkermansia and Bacteroides. TS ameliorated DSS-induced colitis by regulating the gut microbiota, and the gut microbiota could regulate gut inflammation.

Conclusions: These studies demonstrated that TS ameliorated murine colitis through the modulation of immune response, mucus barrier and gut microbiota, thus improving gut dysbiosis. In addition, the gut microbiota may play an important role in regulating the host's innate immune system, and the two coexist and are mutually beneficial. We provide a promising perspective on the clinical treatment of IBD.

Keywords: inflammatory bowel disease, immune response, gut microbiota, intestinal permeability, teasaponin 

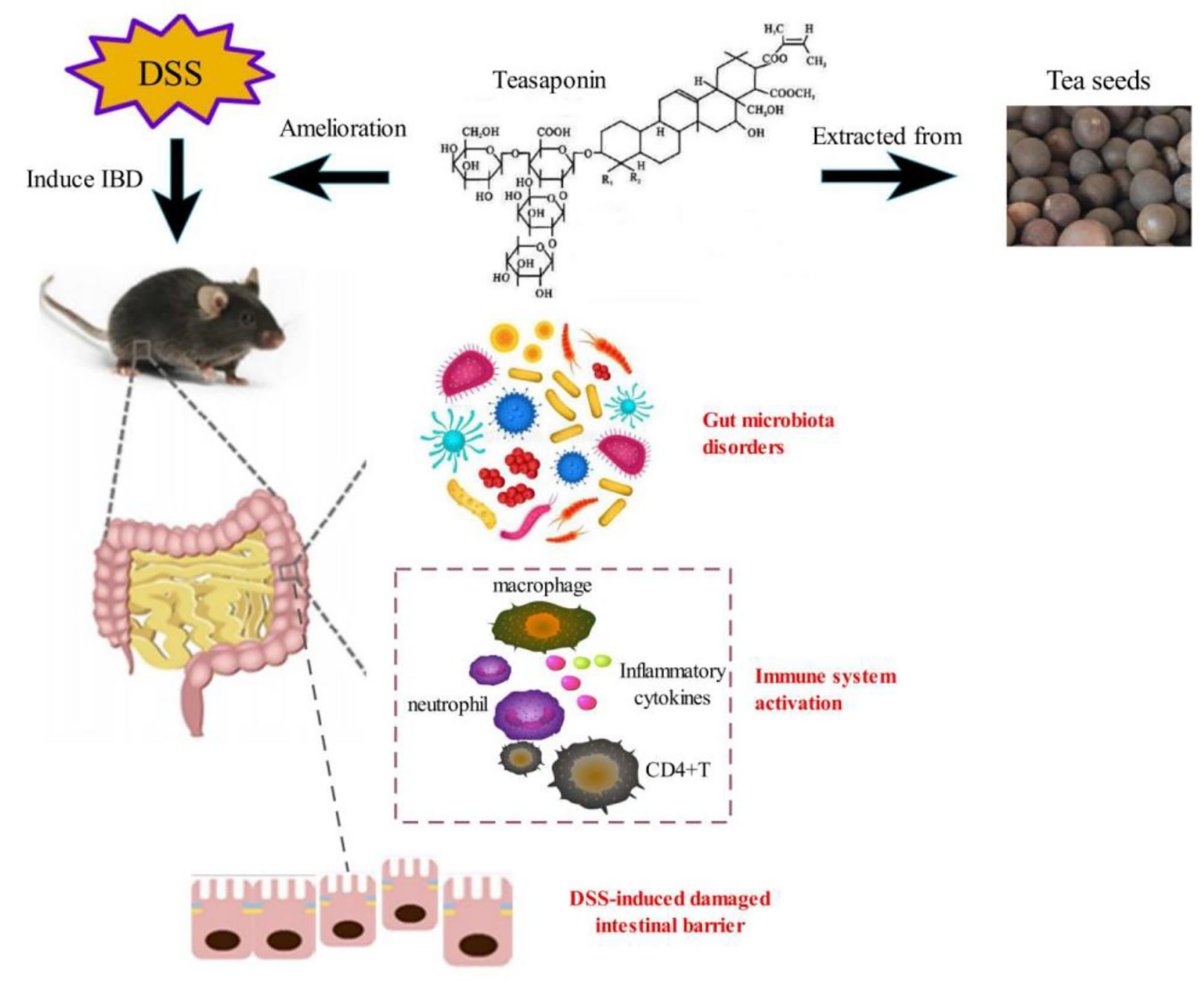

\section{INTRODUCTION}

Ulcerative colitis (UC) is one form of IBD with a substantial impact on the quality of life of affected persons. The incidence of UC is not only high in Western developed countries but on the rise in China and other Asian countries in recent years $(1,2)$. The main symptoms include diarrhea, bellyache, tenesmus and even fever and weight loss. Inflammation is superficial and continuous, confined to the mucosa and submucosa. Notably, UC patients are more likely to develop colorectal cancer (3). There is no determinate conclusion about the pathogenesis of UC, knowing that it may be caused by a combination of host genetics, immune disorders, intestinal microbes, intestinal barrier dysfunction and so on. Numerous clinical data and animal experiments have demonstrated that UC is often accompanied with intestinal immunity dysfunction and imbalance of gut microbiota (4-7).
Common drugs currently available for IBD include aminosalicylates, corticosteroids, thiopurines and biological agents, but they all have serious adverse effects, which urges researchers to develop more effective and less toxic alternative drugs for the treatment of IBD (8). Many studies have focused more attention on natural and functional materials such as polyphenols and terpenoids (7). Terpenoids are natural chemical ingredients with a wide variety of biological activities, especially anti-inflammatory and anticancer potential (9).

Tea has been cultivated for thousands of years and used for beverage and medicinal purposes. Recently, research on tea has shown that tea possesses anti-inflammatory properties, and can improve microbiota and cognition (10). TS is major active component of tea, chemically belonging to oleanane-type pentacyclic triterpene saponins (11). TS has anti-inflammatory, analgesic, anti-osmotic and other pharmacological activities. 
Different mechanisms have been proposed to participate in the beneficial effects of TS, such as reducing inflammatory cytokines by inhibiting the main inflammatory regulators including NF- $\kappa$, which is known to play a crucial role in the pathogenesis of IBD (11). Wang et al. also demonstrated that TS could reduce highfat (HF)-induced endotoxemia and macrophage accumulation in the colon. In addition, TS could improve the gut microbiota alteration caused by HF diet and limit the growth of unfavorable gut microbiota (10).

However, little information about the effect of TS on IBD is available. We aimed to investigate the intestinal antiinflammatory activities and the effect on the gut microbiota of TS in a DSS-induced colitis mouse model. It was found that the effect was associated with decreased immune response and inflammatory cytokines. In addition, the effect on the gut microbiota demonstrated that interventions targeting the microbiota are a potential path for IBD treatment. Our results suggested that TS-associated microbiota was consistent with immune response, and proved that TS may be a potential therapeutic target for patients with IBD.

\section{METHODS}

\section{Establishment of the DSS-Induced Colitis Mouse Model}

All animal experiments were performed according to the standards in the Guide for the Care and Use of Laboratory Animals (Institute of Laboratory Animal Resources of National Research Council, United States). All mouse studies were evaluated by the Laboratory Animal Ethics Committee of Xuzhou Medical University (IACUC number: 201908A005), Xuzhou, China. We made every effort to minimize animal suffering and to reduce animals used.

Male C57BL/6J mice aged 6-8 weeks were purchased from Vital River Laboratory Animal Technology Co., Ltd. (Zhejiang, China). All mice were housed under specific pathogen-free conditions at the Experimental Animal Center of Xuzhou Medical University (Xuzhou, China).

The mice were randomly divided into three different groups: NC group, DSS group, and TS treatment (DSS+TS) group. Animal in NC and DSS group received sterile water $(100 \mu \mathrm{L} /$ day $)$ orally, and those in DSS+TS group received TS (400 mg/kg/day) for 16 days by oral gavage. At day 8 , colitis was induced by $2 \%$ DSS (36-50 kDa, MP Biomedicals, Canada) in the drinking water for 8 days. Teasaponin is extracted from Camellia oleifera seeds. Teasaponin belongs to the triterpene saponins. It has strong foaming, emulsifying, dispersing and wetting effects. Teasaponin $\left(60 \% \geq, \mathrm{C}_{57} \mathrm{H}_{90} \mathrm{O}_{26}\right.$, molecular weight 1,200$)$ was purchased from the Aladdin Chemistry Co., Ltd. (Shanghai, China).

The score of DAI was calculated by body weight, the presence of blood in the feces and stool consistency. The following scores were given: (i) weight loss (0: no loss, 1: 1-5\%, 2: 5-10\%, 3: 10-20\%, 4:>20\%), (ii) bleeding (0: no blood, 1: hemoccult + , 2: hemoccult + and visual pellet bleeding, 4: gross bleeding with blood seen around the anus); and (iii) stool consistency (0: normal, 2: loose stool, 4: diarrhea). On day 16, the mice were sacrificed and the colon tissue and cecal contents were collected. The middle of the colon was fixed in $4 \%$ formaldehyde for histological observation. The remaining colon and cecal contents were stored at $-80^{\circ} \mathrm{C}$.

\section{Enzyme-Linked Immunosorbent Assays (ELISA)}

The upper colon tissue was weighed, homogenated with phosphate buffer solution and centrifuged at 10,000 $\mathrm{rpm} / \mathrm{min}$ for $10 \mathrm{~min}$. The extracted total protein in the supernatant was collected to measure the expression of TNF- $\alpha$, IL- $1 \beta$, IL-6, IL17, and IL-33 by ELISA kits (Neobioscience, Tianjin, China), according to the manufacturer's instructions. The results were read with a microplate reader (Bio-Rad iMARK, USA) at $450 \mathrm{~nm}$.

\section{RNA Extraction and RT-PCR}

The total RNA from the lower colon was extracted using the Total RNA Extraction Kit (Solarbio, Beijing, China) according to the manufacturer's protocol and purified with lithium chloride described by Viennois et al. (12). mRNA was quantified with UltraSYBR Mixture (Cwbio, Beijing, China) and specific primers by qPCR (Roche Light Cycler 480 II, USA) according to the protocol of the manufacturer. Gene expression was normalized to $\beta$-actin and calculated by $2^{-\Delta \Delta \mathrm{Ct}}$. The primers were: $\beta$-actin: $5^{\prime}$ GGCTGTATTCCCCTCCATCG3' and 5'CCAG TTGGTAACAATGCCATGT3'; TNF- $\alpha$ : 5'GA TCGGTCCCCA AAGGGATG3' and 5'TTTGCTACGACGTGGGCTAC3'; IL1 $\beta$ : 5'TGCC ACCTTTTGACAGTGATG3' and 5'ATGTGCTG CTGCGAGATTTG3'; IL-6: 5'AGACAA AGCCAGAGTCCT TCAG3' ${ }^{\prime}$ and 5'GAGCATTGGAAATTGGGGTAGG3'; IL-17: 5' TTTA ACTCCCTTGGCGCAAAA $3^{\prime}$ and 5'CTTTCCCTCCGC ATTGACAC3'; IL-33: 5' TCCAACTCCAAGATTTCCCCG3' and 5'CATGCAGTAGACATGGCAGAA3'.

\section{Histological and Morphometric Analysis}

The middle of the colon was fixed in $4 \%$ formaldehyde. Histological sections $(4 \mu \mathrm{m})$ were stained with $\mathrm{HE}$ for morphometric examination. The score was according to the degree of inflammatory infiltration (0-5), crypt injury (0-4), ulcer ( $0-3)$, and the absence of edema ( 0 or 1$)$, as described by Stillie et al. (13).

The slides were deparaffinized and then incubated with F4/80 antibody (Abcam, 1:100), Ly6G antibody (Abcam, 1:100), CD4 antibody (Servicebi, 1:100), Muc2 antibody (Proteintech, 1:100), Claudin-1 antibody (Proteintech, 1:100) and ZO-1 antibody (Proteintech, 1:100) in blocking buffer overnight at $4^{\circ} \mathrm{C}$ overnight, followed by incubation with Alexa Fluor 594labeled second antibody (Proteintech) for $1 \mathrm{~h}$. The sections were then stained with DAPI for nuclear counterstaining. The images were observed by fluorescence microscopy (Olympus IX71, Tokyo, Japan).

The slides were deparaffinized, stained with Phospho-NF-кB p65 (Servicebio, 1:100) and Phospho-STAT3 antibody (Abcam, 1:100) overnight at $4^{\circ} \mathrm{C}$, incubated with a rabbit HRP polyclonal antibody for $1 \mathrm{~h}$ at room temperature, and finally visualized with DAB (VECTOR). 


\section{Microbial Community Analysis by $16 \mathrm{~S}$ rRNA Gene Sequencing}

Mice cecal contents were collected and stored at $-80^{\circ} \mathrm{C}$ after snapping frozen in liquid nitrogen. The DNA of total bacteria in mice cecal contents was extracted with QIAamp ${ }^{\circledR}$ Fast DNA Stool Mini Kit. For 16S rRNA gene sequencing, the DNA samples were sent to Microbiology Division, Meiji Biomedical Technology Co., Ltd. (Shanghai, China) under $-20^{\circ} \mathrm{C}$ preservation and dry ice conditions. High fidelity PCR was utilized to amplify bacterial $16 \mathrm{~S}$ rRNA hypervariable region 3 (V3) and hypervariable region 4 (V4) with the primers. High-throughput sequencing was performed on Illumina Miseq platform with $2 \times 250$ bp paired-end method after quantifying, mixing and quality checking the library. All the results were based on sequenced reads and operational taxonomic units (OTUs).

\section{FITC-dextran Assay for Detecting Intestinal Permeability}

On the 16th day of the TS treatment, the mice were deprived of water for $2 \mathrm{~h}$, and then each mouse was given a $40 \mathrm{mg} / 100 \mathrm{~g}$ FITC-dextran solution. After intragastric administration for $4 \mathrm{~h}$, blood was taken from the mouse eyeballs, centrifuged at $4^{\circ} \mathrm{C}, 3,000 \mathrm{rpm} / \mathrm{min}$ for $15 \mathrm{~min}$, and serum was obtained. The fluorescence intensity of FITC was detected at 485/530 nm with a fluorescence microplate reader, and the concentration of FITC-dextran in the sample was calculated according to the standard curve.

\section{Fecal Microbiota Transplantation}

First, mice are randomly divided into donor group and recipient group. For donor group, animal in NC and DSS group received sterile water $(100 \mu \mathrm{L} /$ day $)$ orally, and those in DSS+TS group received TS (400 mg/kg/day) for 16 days by oral gavage. At day 8 , colitis was induced by $2 \%$ DSS in the drinking water for 8 days. At day 10, in order to establish a sterile mouse model, recipient mice were given a mixture of antibiotics (ampicillin $1 \mathrm{mg} / \mathrm{mL}$, neomycin $1 \mathrm{mg} / \mathrm{mL}$, metronidazole $1 \mathrm{mg} / \mathrm{mL}$ and vancomycin $0.5 \mathrm{mg} / \mathrm{mL})$ in drinking water and oral gavage $(200 \mu \mathrm{L} /$ day $)$ for 5 days. At day 15, stopped antibiotics and prepared fecal microbiota transplantation. At day 16, $200 \mathrm{mg}$ per mouse of feces from donor mice was dissolved in $1 \mathrm{ml}$ of $0.05 \%$ cysteine solution, stirring well and filtering with $100 \mu \mathrm{l}$ screen to obtain fresh fecal filtrate, which was administered to recipient mice by orally gavage (200 $\mu \mathrm{L} /$ mouse) for 5 consecutive days. Body weight and DAI were observed every day, and feces were collected to test the colonization efficiency of fecal microbiota.

\section{The Detection of Fecal Lipocalin 2}

The feces were weighed, homogenated with phosphate buffer solution and centrifuged at $10,000 \mathrm{rpm} / \mathrm{min}$ for $10 \mathrm{~min}$. The extracted total protein in the supernatant was collected to measure the expression of lipocalin 2 (LCN2) by ELISA kits (Abcam), according to the manufacturer's instructions. The results were read with a microplate reader (Bio-Rad iMARK, USA) at $450 \mathrm{~nm}$.

\section{The Myeloperoxidase Assay in Tissues}

The upper colon tissue was weighed, homogenated with phosphate buffer solution and centrifuged at $10,000 \mathrm{rpm} / \mathrm{min}$ for $10 \mathrm{~min}$. The extracted total protein in the supernatant was collected to measure the expression of Myeloperoxidase (MPO) by ELISA kits (Abcam), according to the manufacturer's instructions. The results were read with a microplate reader (Bio-Rad iMARK, USA) at $450 \mathrm{~nm}$.

\section{Flow Cytometry Analysis of Colon Tissues}

The colon tissues were sliced with a scalpel into many small pieces and digested for $30 \mathrm{~min}$ at $37^{\circ} \mathrm{C}$ with $0.5 \mathrm{mg} / \mathrm{ml}$ collagenase (Sigma-Aldrich) and $100 \mathrm{~g} / \mathrm{ml}$ DNAse I in PBS. After digestion, tissues were mashed and single cell suspensions were filtered through a $70 \mu \mathrm{m}$ nylon mesh and washed with PBS. Then we separated mononuclear cells with $40 \%$ percoll. Single cell suspensions were incubated with following antibodies: anti-CD11b conjugated to APC (eBioscience, San Diego, CA, USA), anti-Ly6G conjugated to PE (eBioscience), anti-F4/80 conjugated to FITC (eBioscience, San Diego, CA, USA), antiCD45 conjugated to APC (eBioscience, San Diego, CA, USA), anti-CD4 conjugated to $\mathrm{PE}$ (eBioscience), anti-CD3 conjugated to FITC (eBioscience), Cells were analyzed on a FACSCanto II Flow Cytometer (BD Biosciences) using the Flow Jo software (FlowJo, Ashland, OR, USA).

\section{Statistical Analysis}

Statistical analysis was performed using GraphPad Prism (GraphPad Software). Data are expressed as the mean \pm SEM. Comparisons between two groups were assessed using a student $t$-test or Mann-Whitney test depending on whether the data were normally distributed. Statistical significance between multiple groups was tested using a one-way multiple analysis of variance (ANOVA) or Kruskal-Wallis. The level of statistical significance was set at $p<0.05$.

\section{RESULTS}

\section{TS Suppressed DSS-Induced Colitis in Mice}

To clarify the role of TS in DSS-induced colitis, we treated the mice with TS for 16 days and induced murine DSS colitis by administrating $2 \%$ DSS in drinking water from day 8 (Figure 1A). The administration of 2\% DSS for 8 days produced an increase in DAI scores, due to decreased body weight and diarrheic/bleeding feces. However, the mice treated with TS showed a reduced DSS damage, including body weight loss and a lower incidence of diarrheic/bleeding feces (Figures 1B,C), resulting in lower DAI scores in the DSS+TS group compared with the DSS group (Figure 1D). In addition, the macroscopic observation of the colonic segments demonstrated the ameliorative effect of the DSS+TS group. TS prevents colon shortening compared with that of the DSS group (Figure 1E). 
A

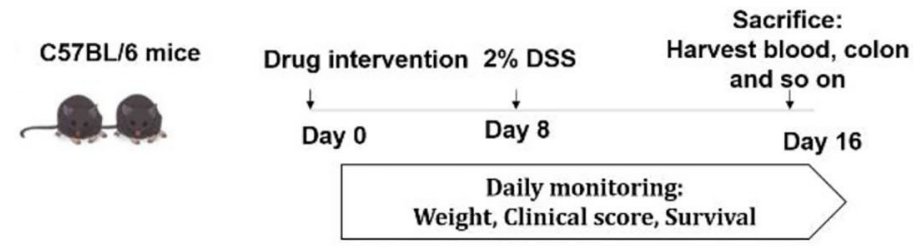

C

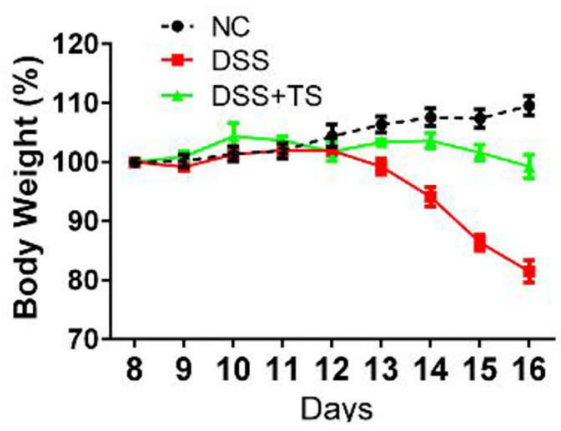

E

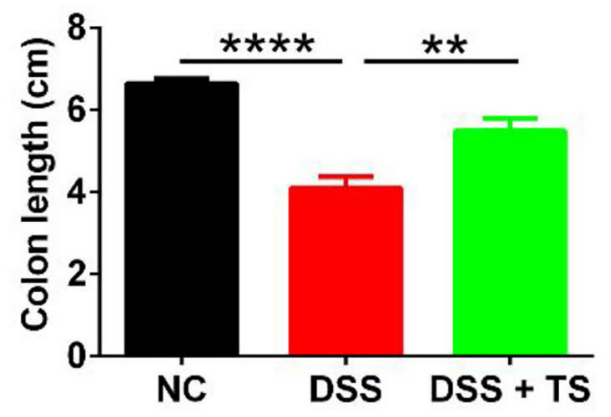

B

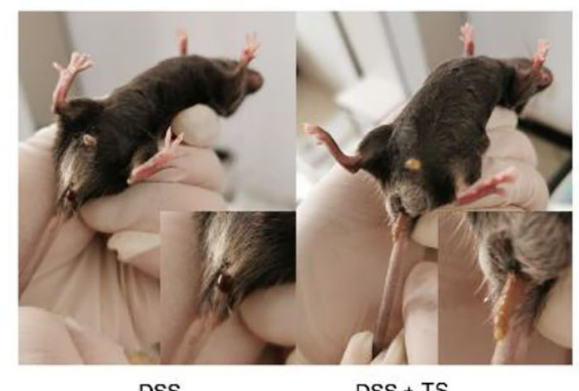

D

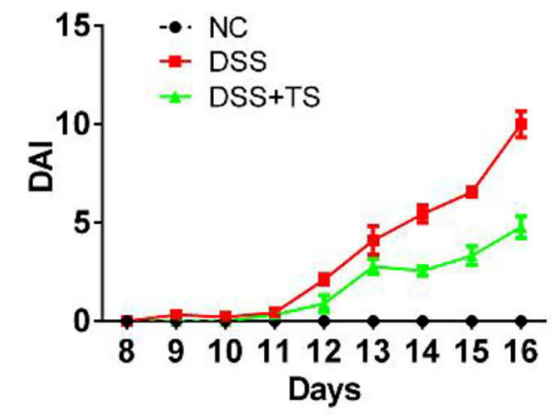

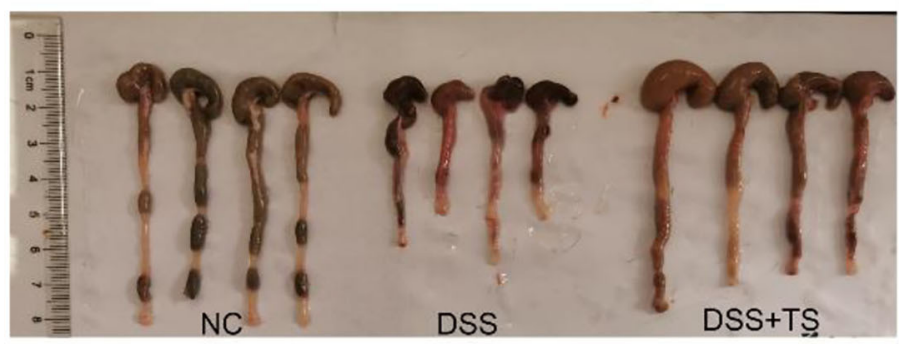

FIGURE 1 | TS ameliorates DSS-induced colitis in mice. (A) Flow diagram; (B) symptoms and fecal characters; (C) body weight; (D) DAl score; (E) colon length. Data are expressed as the mean \pm SEM $(n=9)$. Statistical significance was determined with one-way ANOVA followed by Tukey test, and $p$-values are as follows: ${ }^{\star *} p<$ $0.01,{ }^{\star \star \star \star} p<0.0001$

\section{TS Improved the Colonic Mucus Barrier}

To evaluate the level of the colonic damage under the microscope, the histopathological scores of the representative H\&E stained histological sections were measured (Figure 2A). The colon of the DSS group showed moderate-severe edema and inflammation in the mucosal and muscular layer. Besides, there were more than two-thirds of disappeared crypts and incomplete epithelial cells in the DSS group. The DSS+TS group showed mild edema and inflammation, and the crypts disappeared by about one third. The histological score manifested a significant remission in the DSS+TS group compared with the DSS group.

To further characterize the damage to the epithelial layer, intestinal permeability was quantified by oral administration of FITC-dextran to mice on day 8 of DSS treatment, and fluorescence intensity in the serum was measured (Figure 2B). The diffusion of FITC-dextran from the intestine to the serum after TS treatment was significantly decreased compared with the DSS group, suggesting that TS improved the ability of maintaining the function of the colonic mucus barrier during DSS administration.

Knowing that another crucial component of maintaining the function of the intestinal mucus barrier is the formation and distribution of some proteins between epithelial cells including mucins and tight junction proteins, we observed the expression and distribution of these proteins in the colon, and the results of immunofluorescence analysis of MUC2, ZO-1, and Caludin1 were shown (Figures 2C-E). In the NC group, crypts were filled with MUC2 except the basilar part of the mucosal layer. The distribution of MUC2 was narrowed along with the disappearance of crypts in the DSS group, manifesting a severe colonic damage induced by DSS. While, the distribution of MUC2 in the DSS+TS group was across the mucosal layer, suggesting the compensatory reaction to the slight inflammation. Claudin-1 and ZO-1, both known as the tight junction proteins, 
A
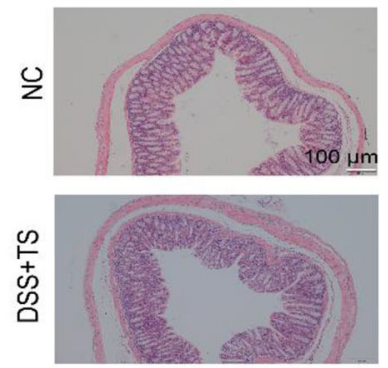

C

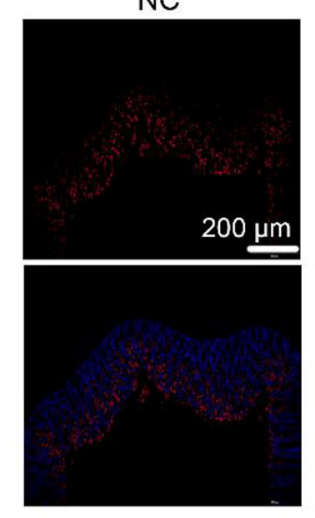

NC

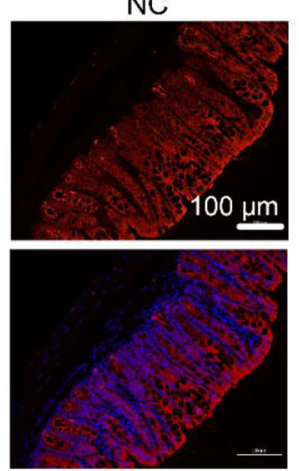

E

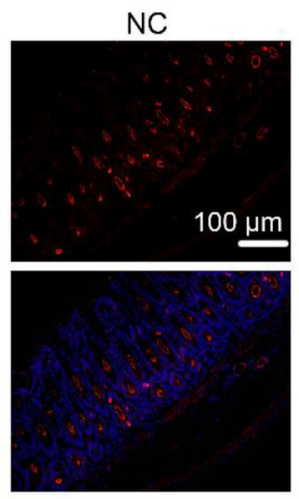

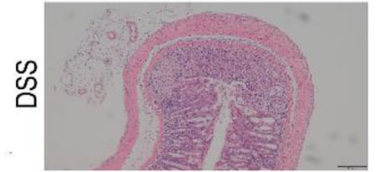

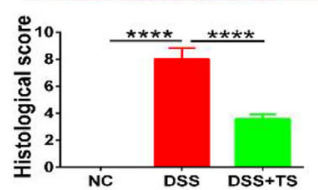

B

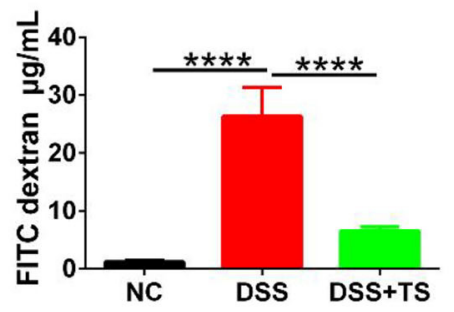

DSS

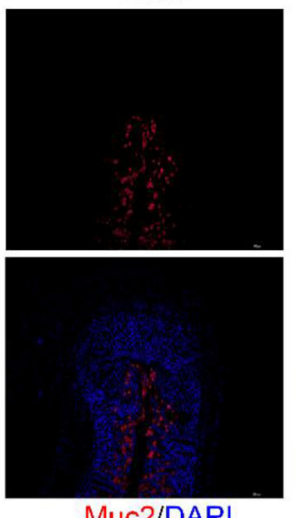

Muc2/DAPI

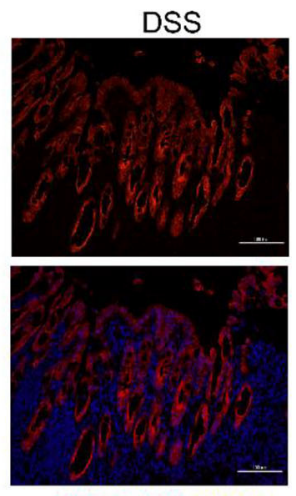

Claudin-1/DAPI
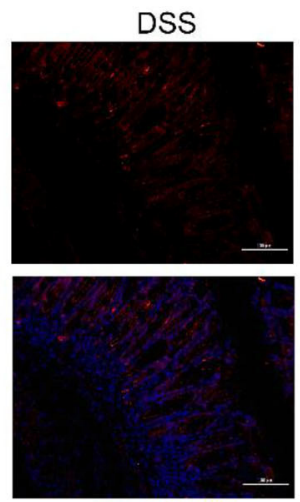

ZO-1/DAPI
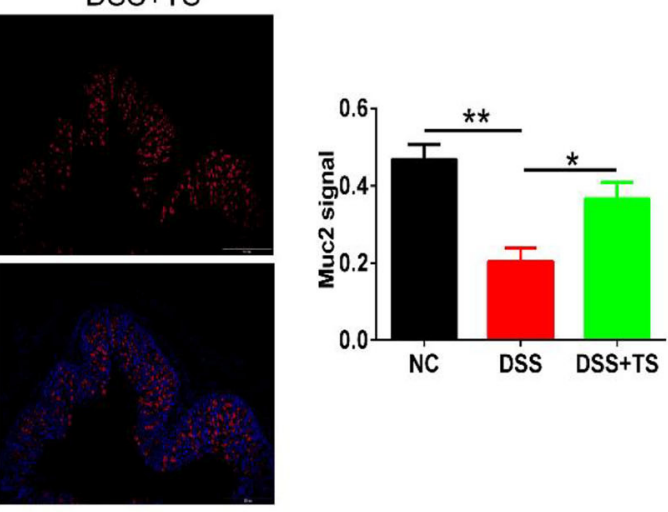

DSS+TS
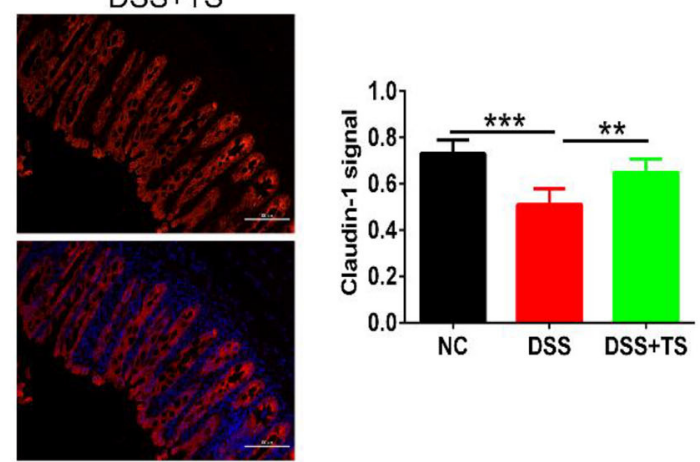

DSS+TS
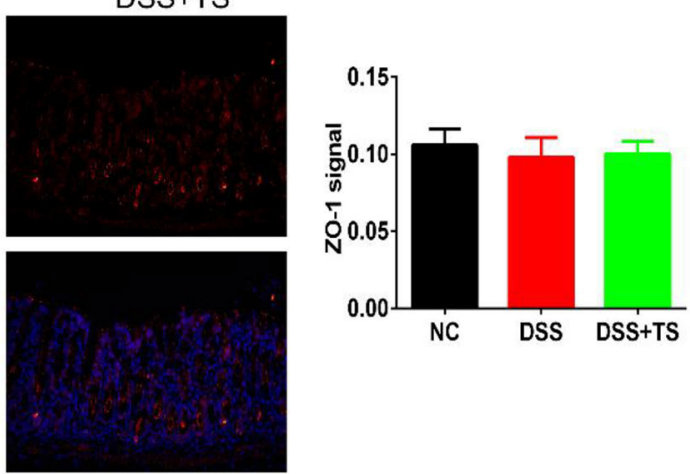

FIGURE 2 | The effect of TS on the mucus barrier damaged by DSS. (A) Representative images of H\&E stained histological sections and histopathology scores; (B) detection of FITC-dextran in serum; (C-E) representative images of immunofluorescence of MUC2, Claudin-1, and ZO-1. The mucins and tight junction proteins are stained red, and nuclei are counterstained blue. Quantitative analysis of Muc2, Claudin-1, and ZO-1 signal was using Image-Pro Plus. Data are expressed as mean \pm SEM $(n=7)$, statistical significance was determined with one-way ANOVA followed by Tukey test, and $p$-values are as follows: ${ }^{\star} p<0.05,{ }^{* \star} p<0.01$, ${ }^{\star \star \star} p<0.001$, ${ }^{\star * \star \star} p<0.0001$. 
were arrayed compactly and orderly in the NC group. The distribution of Claudin-1 and ZO-1 was dispersed and hollow in the DSS group. Conversely, TS improved the damage induced by DSS administration.

\section{TS Attenuated Intestinal Inflammation}

To investigate whether TS resulted in the changes in inflammatory response, levels of TNF- $\alpha$, IL-1 $\beta$, and IL6 were quantified in the colon tissue using RT-qPCR and ELISA. Compared with the NC group, the mRNA levels of these pro-inflammatory cytokines were increased markedly in the DSS group. While the mRNA levels of these observed cytokines were significantly reduced in DSS+TS group (Figures 3A-C). Meanwhile, the protein levels of these inflammatory cytokines manifested the same results (Figures 3D-I). As a frequently used marker to measure DSS induced intestinal inflammation, we inclued the fecal lipocalin 2 ELISA data. The fecal lipocalin 2 showed a significant decrease in the DSS+TS group compared with the DSS group in Figure 3K. Generally, TS reduced the levels of pro-inflammatory cytokines in the colon treated with DSS. Immunohistochemistry (IHC) staining analysis demonstrated that phospho-STAT3 existed in the DSS group, especially localized the place with severe inflammation. In contrast, phospho-STAT3 was not detected in the DSS+TS group (Figure 3J). Here we focused on phospho-P65 (NF-kB signaling), which is associated with the development and progression of inflammation. IHC staining showed that TS inhibited P65 phosphorylation level relative to the DSS group (Figure 3K).

\section{TS Ameliorated Immune System Disorder Induced by DSS}

Elevated levels of inflammatory cytokines are markers of immune-related disorder. In general, the release of inflammatory factors recruits immune cells. Therefore, we investigated relevant immune cells in the colon tissue by immunofluorescence (Figures 4A-C) and flow cytometry analysis (Figures 5A-C). Ly6G, a marker for neutrophils, was increased in the DSS group, but the TS reduced the recruitment. We used the CD45 marker to separate white blood cells first, and CD3CD4 double positive represents $\mathrm{CD} 4+\mathrm{T}$ cells. $\mathrm{CD} 3+\mathrm{CD} 4+\mathrm{T}$ cells were also increased in the DSS group compared with the other groups. The expression was decreased in the DSS+TS group, even though the difference was not statistically significant. F4/80 is a marker for macrophages, which gives rise to pro-inflammatory cytokines. It was elevated in the DSS group compared with the other groups. The expression was reduced in the DSS+TS group, but the difference was not statistically significant. Therefore, TS mainly played a role in recruiting fewer neutrophils to reduce inflammation.

\section{Impacts of TS on the Gut Microbiota in DSS-Induced Colitis}

To determine the impact of TS on the gut microbiota in DSS-induced colitis, we detected the gut microbiota by 16s rRNA sequencing. There was no significant difference in the OTU number between the NC, DSS, and DSS+TS groups (Figure 6A). The $\beta$-diversity analysis showed a distinct demarcation in microbial composition between the three groups, including the principal components analysis (PCA), principal coordinate analysis $(\mathrm{PCoA})$ and the system clustering tree (Figures 6B-D). The PCA (ANOSIM: $R=0.3209, p$ $=0.0010)$ and PCoA (ANOSIM: $R=0.7716, p=0.0010$ ) of Bray-Curtis based on OTUs presented that DSS changed the gut microbiota significantly, while TS could regulate the gut microbiota (Figures 6B,C). The system clustering tree demonstrated the same result that DSS+TS group was clustered separately from DSS group (Figure 6D). However, TS only changed some microbiome and had little effect on the composition of total gut microbiota, so DSS-TS microbiome was more similar to the DSS group than the NC group.

Microbial community histogram disclosed the compositions of the intestinal microbiota at a phylum level (Figures 7A,B). The detected phyla included Firmicutes, Bacteroidetes, Proteobacteria, Actinobacteria, Verrucomicrobia, and Deferribacteres. In the DSS group, the abundance of Bacteroidetes was decreased compared with the NC group. The modification in Bacteroidetes also resulted in an increase in the $\mathrm{F} / \mathrm{B}$ ratio in the DSS group compared with the NC group. While the abundance of Bacteroidetes was increased and the F/B ratio was reduced in DSS+TS group. We also noted Actinobacteria, which was significantly more abundant in the NC group, than in the DSS and DSS+TS groups. The linear discriminant analysis effect size (LEfSe) method was employed to identify the characteristic bacterial taxa among these groups (Figures 7C,D). In the cladogram, the key bacterial taxa for each group was marked with relevant colorful nodes at the levels of phylum, class, order, family and genus (Figure 7C). The prominent bacterial taxa for each group was also displayed according to the LDA scores in the LEfSe Bar (Figure 7D). The results demonstrated that the predominant bacteria in the DSS group contained several pathogens like Campylobacteria, Proteobacteria, Helicobacter, and Enterobacteriaceae. Additionally, some probiotics like Akkermansia, Bacteroides, and Lactobacillus were increased in DSS+TS groups compared with DSS group, suggesting the positive effect of TS on the intestinal microbiota.

\section{TS Treatment Changed the Metabolic Genes}

Besides the microbiota community, the discrepancy of functional profiles between different groups predicted by PICRUSt software is exhibited in Figure 8. The investigation of communities by PICRUSt was used in our work and the predicted metagenome information was then collapsed into the KEGG pathway (level 3) based on the 16S rRNA sequences. SangerBox package was used for functional profiling. In total, the treatment of DSS mainly caused changes in 6 pathways (Figure 8A), including the circulatory system, p53 signaling pathway, NOD-like receptor signaling pathway, 
A

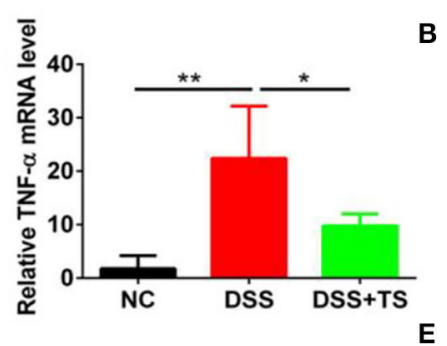

D

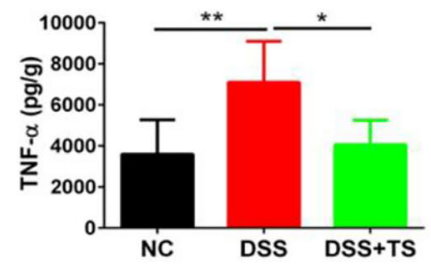

G

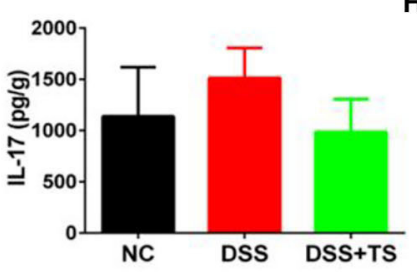

J

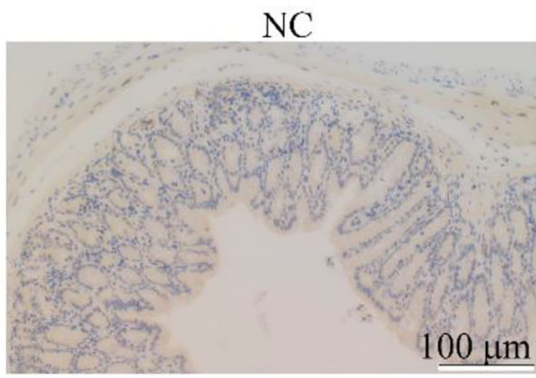

$\mathbf{K}$

$\mathrm{NC}$

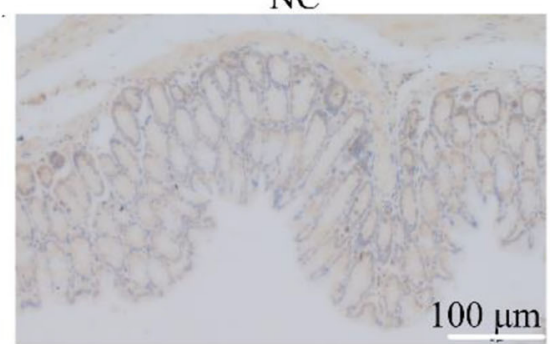

H

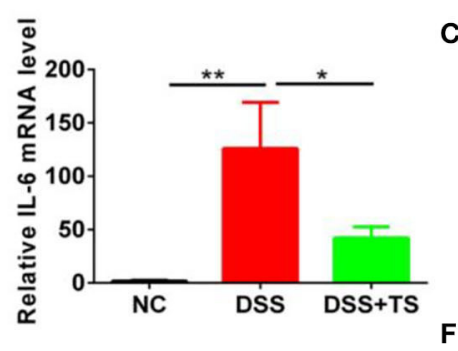

C
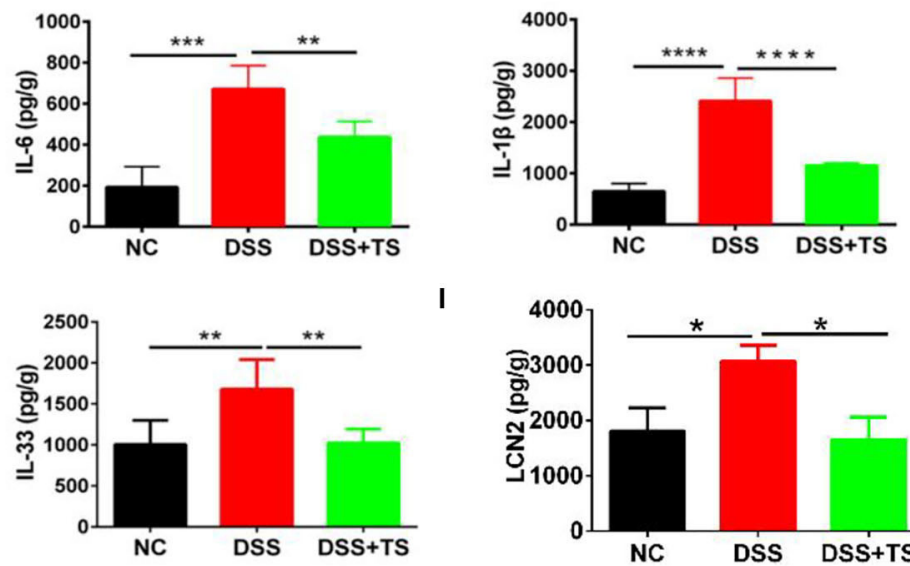

DSS

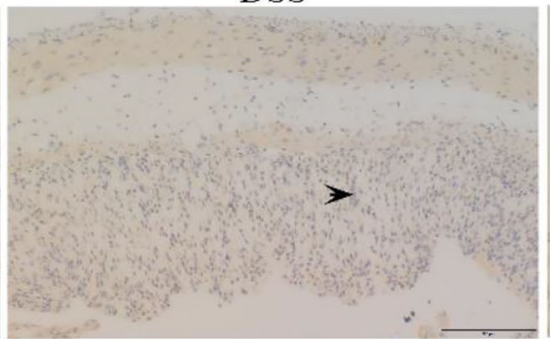

DSS

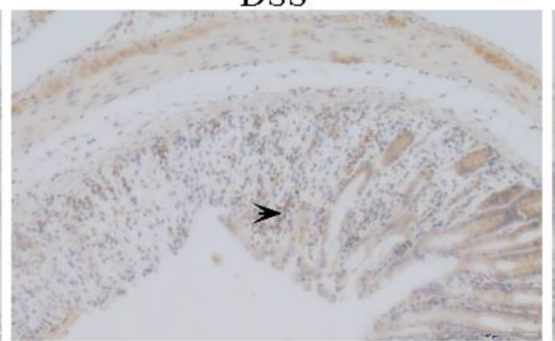

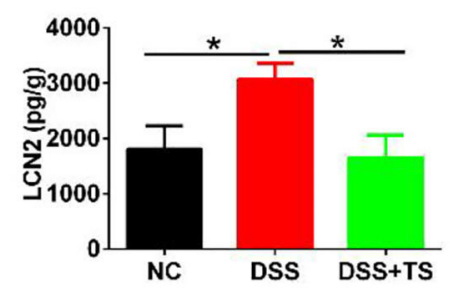

DSS+TS
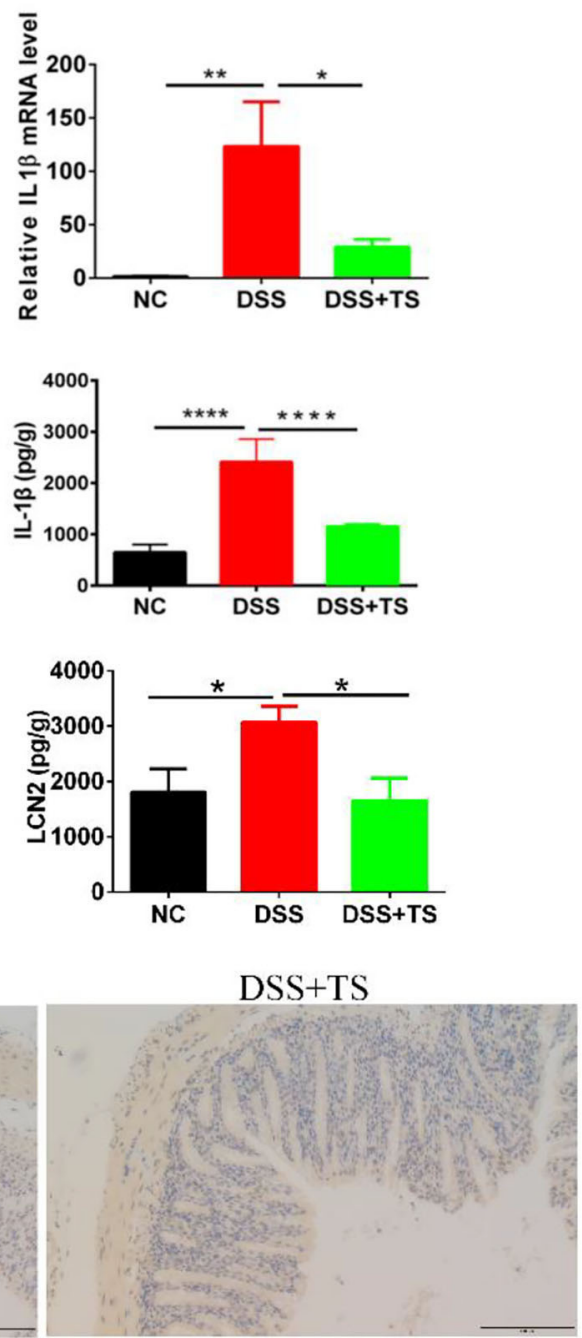

DSS+TS

FIGURE 3 | The effect of TS on the expression of inflammatory signal caused by DSS. (A-C) The mRNA levels of TNF- $\alpha$, IL-6, and IL-1 $\beta$ by RT-qPCR; (D-F) the protein levels of TNF- $\alpha$, IL-6, and IL-1 $\beta$ by ELISA; $(\mathbf{G}, \mathbf{H})$ the protein levels of IL-17 and IL-33 by ELISA; (I) the levels of fecal LCN2 by ELISA; (J) representative immunohistochemistry images of p-STAT3 in the colon tissues. (K) representative immunohistochemistry images of p-P65 in the colon tissues. The arrows indicate the positive staining p-STAT3 and p-P65. Data are expressed as mean \pm SEM $(n=5)$, statistical significance was determined with one-way ANOVA followed by Tukey test, and $p$-values are as follows: ${ }^{*} p<0.05,{ }^{* \star} p<0.01,{ }^{\star \star *} p<0.001,{ }^{\star \star \star \star} p<0.0001$.

flagellar assembly, bacterial invasion of epithelial cells, and bacterial motility proteins. Compared with the NC group, DSS treatment promoted these pathways, while these pathways were down-regulated after TS treatment. Meanwhile, the treatment of DSS caused changes in some genes and the gene abundances in pathways of drug metabolism, tyrosine 
A

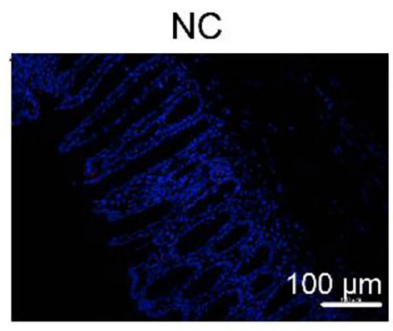

B

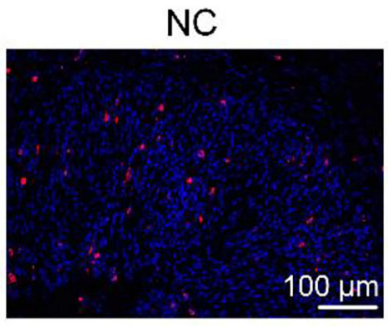

C

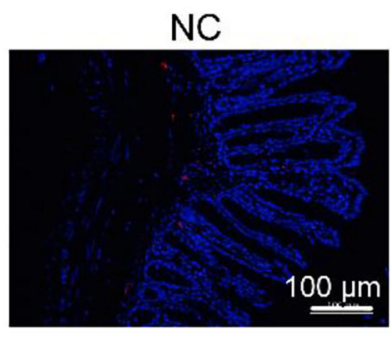

DSS

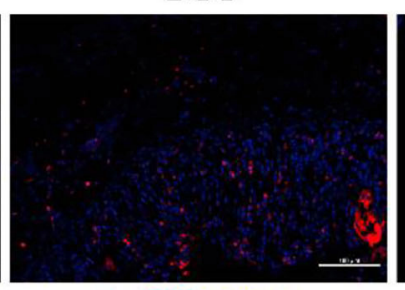

Ly6G/DAPI

DSS

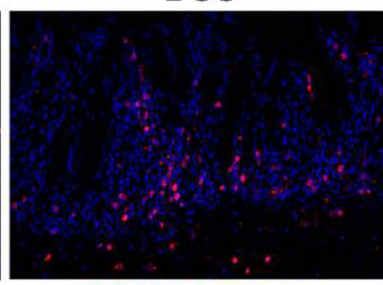

CD4+T/DAPI

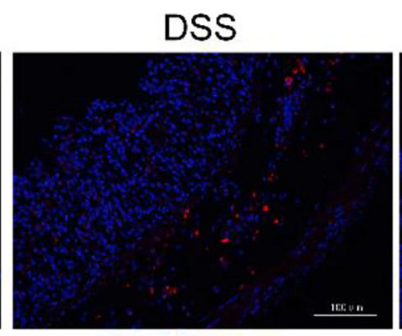

F4/80/DAPI
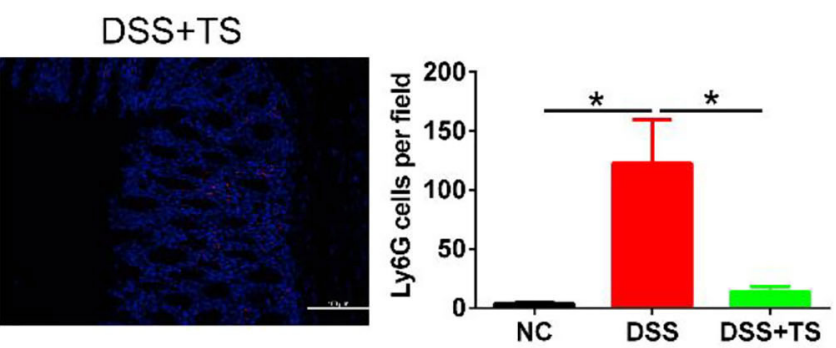

DSS+TS
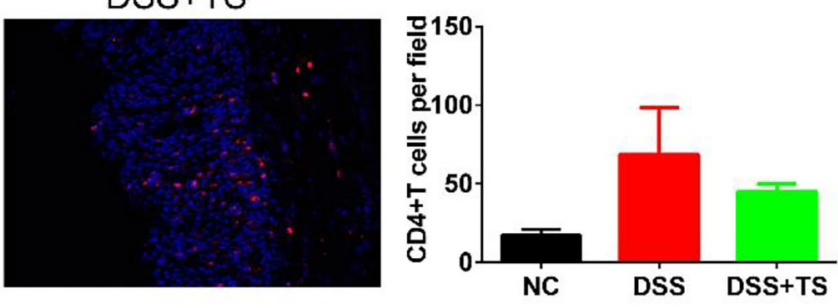

\section{DSS+TS}
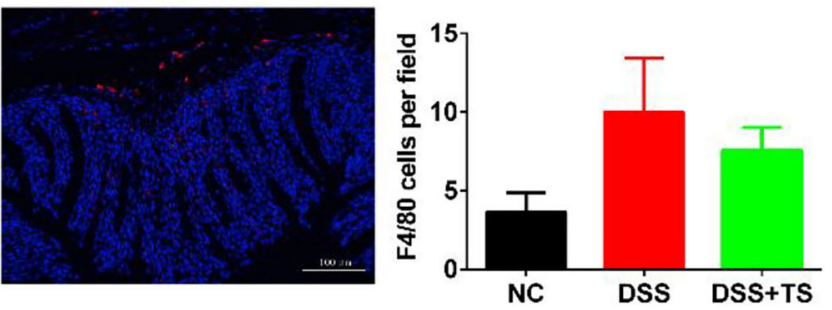

FIGURE 4 | Effects of TS on the immune system disorder analyzed by immunofluorescence. (A) Representative immunofluorescence staining images of neutrophils (Ly6G) and mean positive cells counted per field in the colon tissues $(n=5)$; (B) representative immunofluorescence staining images of T cells $(C D 4+T)$ and mean positive cells counted per field in the colon tissues $(n=5)$; (C) representative immunofluorescence staining images of macrophage (F4/80) and mean positive cells counted per field in the colon tissues $(n=5)$; The immune cells (Ly6G, CD4+T, F4/80) are stained red, and nuclei are counterstained blue. Data are expressed as mean \pm SEM, statistical significance was determined with one-way ANOVA followed by Tukey test, and $p$-values are as follows: ${ }^{*} p<0.05$.

metabolism, glycolysis/gluconeogenesis, fructose and mannose metabolism, melanogenesis, Huntington's disease (Figure 8B). TS treatment led to changes in more complex functional pathways, mainly in fructose and mannose metabolism, pyrimidine metabolism, amino sugar and nucleotide sugar metabolism, ribosome and human diseases such as melanogenesis, Huntington's disease (Figure 8C). Therefore, TS intervention contributed to the functional difference of the gut microbiota.

\section{The Effect of Gut Microbiota Induced by TS in DSS-Induced Colitis in Mice}

To confirm the important role of microbiota induced by TS, we conducted the fecal transfer experiment. We took the feces of the NC group, DSS group and DSS+TS group, and administered them to antibiotic-treated sterile mice by orally gavage for 5 consecutive days. The mice treated with gut microbiota of DSS+TS group showed a reduced DSS damage, including body weight loss and a lower DAI scores in the group that gut microbiota administered by DSS+TS group compared with the DSS group (Figures 9A,B). The general appearance and length of the colon were not significantly different between the DSS group and the DSS+TS group in Figures 9C,D. In addition, the diffusion of FITC-dextran from the intestine to the serum after gut microbiota induced by TS treatment was significantly decreased compared with the DSS group (Figure 9E), suggesting that gut microbiota induced by TS improved the ability of maintaining the function of the colonic mucus barrier during DSS administration.

\section{The Association of Gut Microbiota Induced by TS With Gut Inflammation}

We demonstrated that TS ameliorated murine colitis through the modulation of immune response, mucus barrier and gut microbiota, thus improving gut dysbiosis, However, there was no relation analysis between the immune response 
A

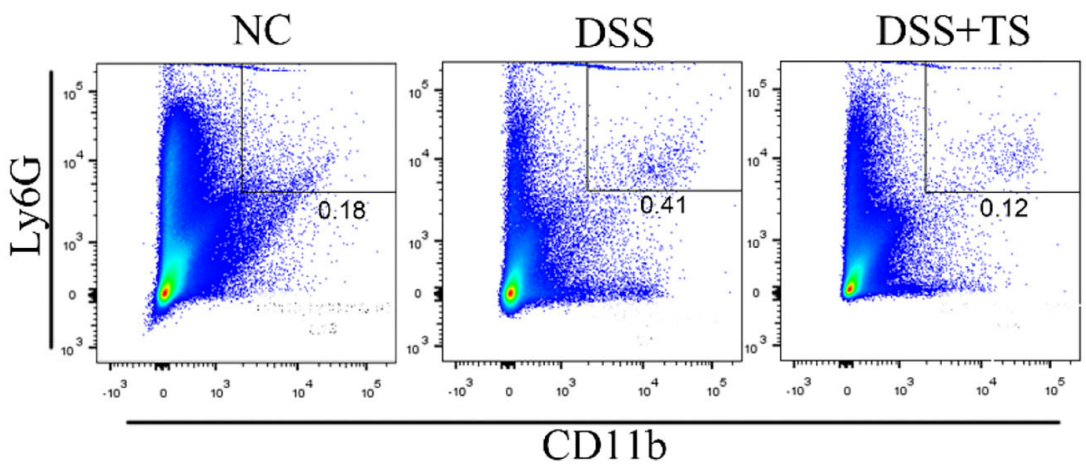

B

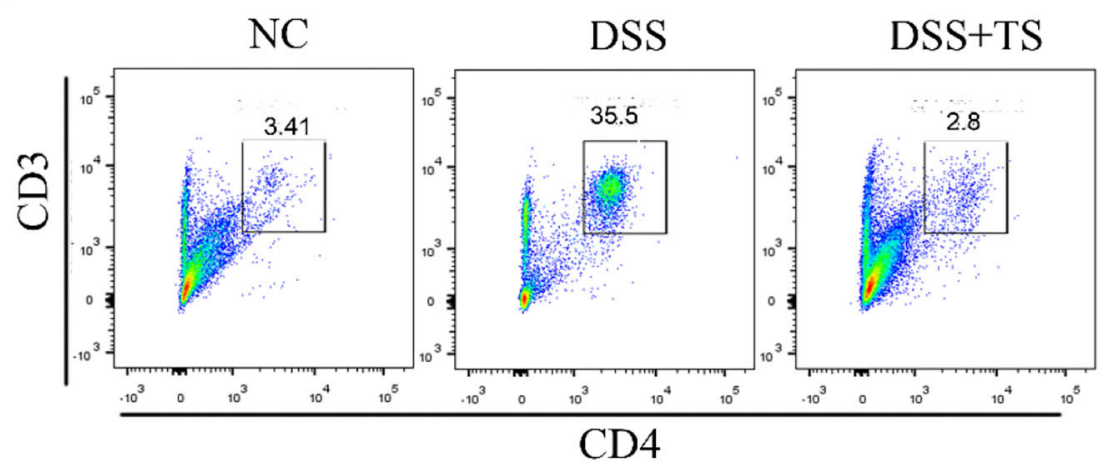

C

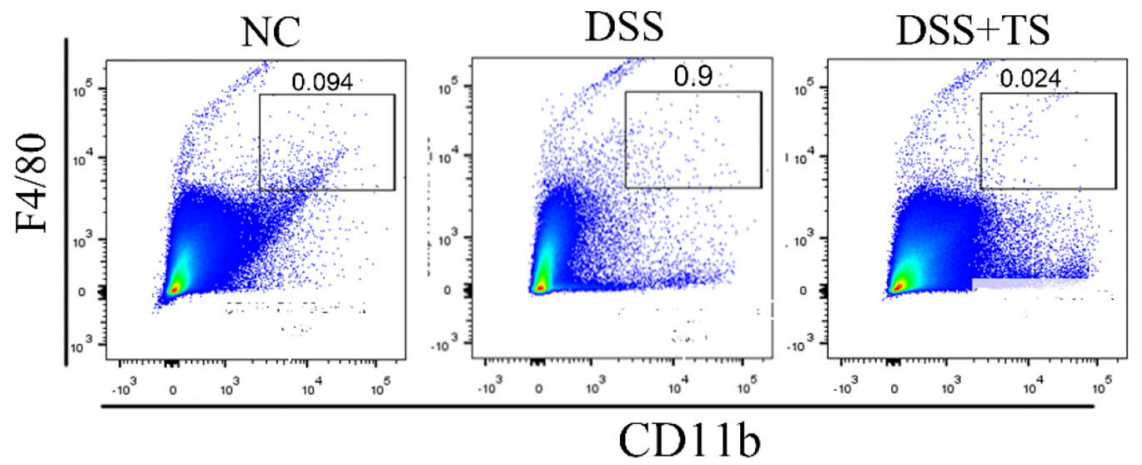

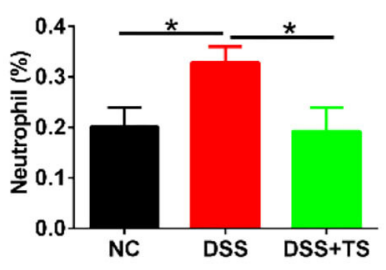
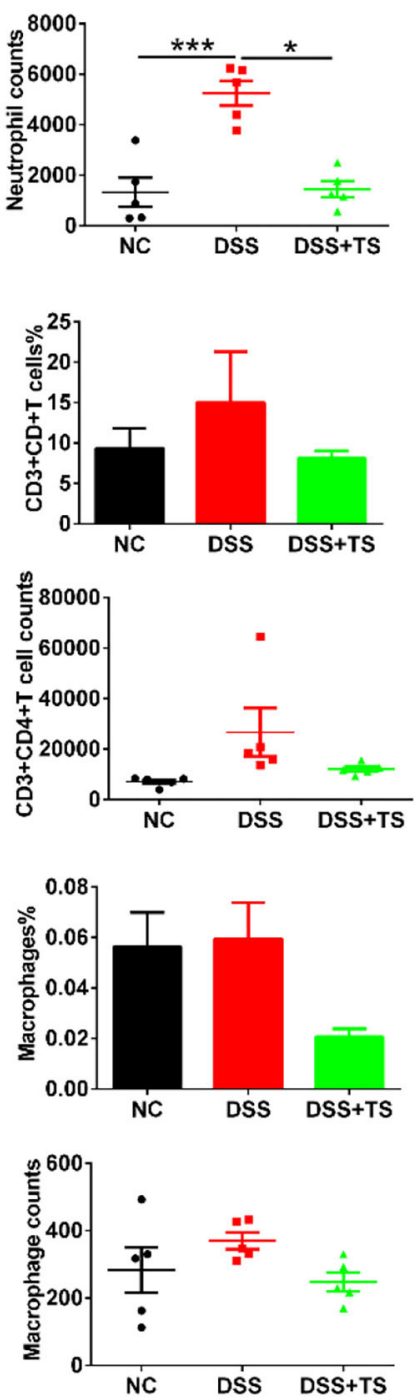

FIGURE 5 | Effects of TS on the immune system disorder analyzed by flow cytometry. Percentages and counts of neutrophils (A), CD4+T cells (B) and macrophages (C) in lamina propria of colon $(n=5)$. Data are expressed as mean \pm SEM, statistical significance was determined with one-way ANOVA followed by Tukey test, and $p$-values are as follows: ${ }^{*} p<0.05,{ }^{* \star *} p<0.001$.

and gut microbiota. We took the feces of the NC group, DSS group and DSS+TS group, and administered them to antibiotic-treated sterile mice. The mice treated with gut microbiota of TS treatment showed a reduced level of IL6 (Figure 10A), TNF- $\alpha$ (Figure 10B), and MPO (Figure 10C) compared with the DSS group, however, TNF- $\alpha$ showed no statistical difference.

\section{DISCUSSION}

DSS treatment was reported to shorten the length of the colon and increased DAI scores including weight loss, diarrhea and hematochezia in mice (14), while TS intervention increased body weight, ameliorated bloody stools, increased the colon length and decreased the DAI score, indicating that 


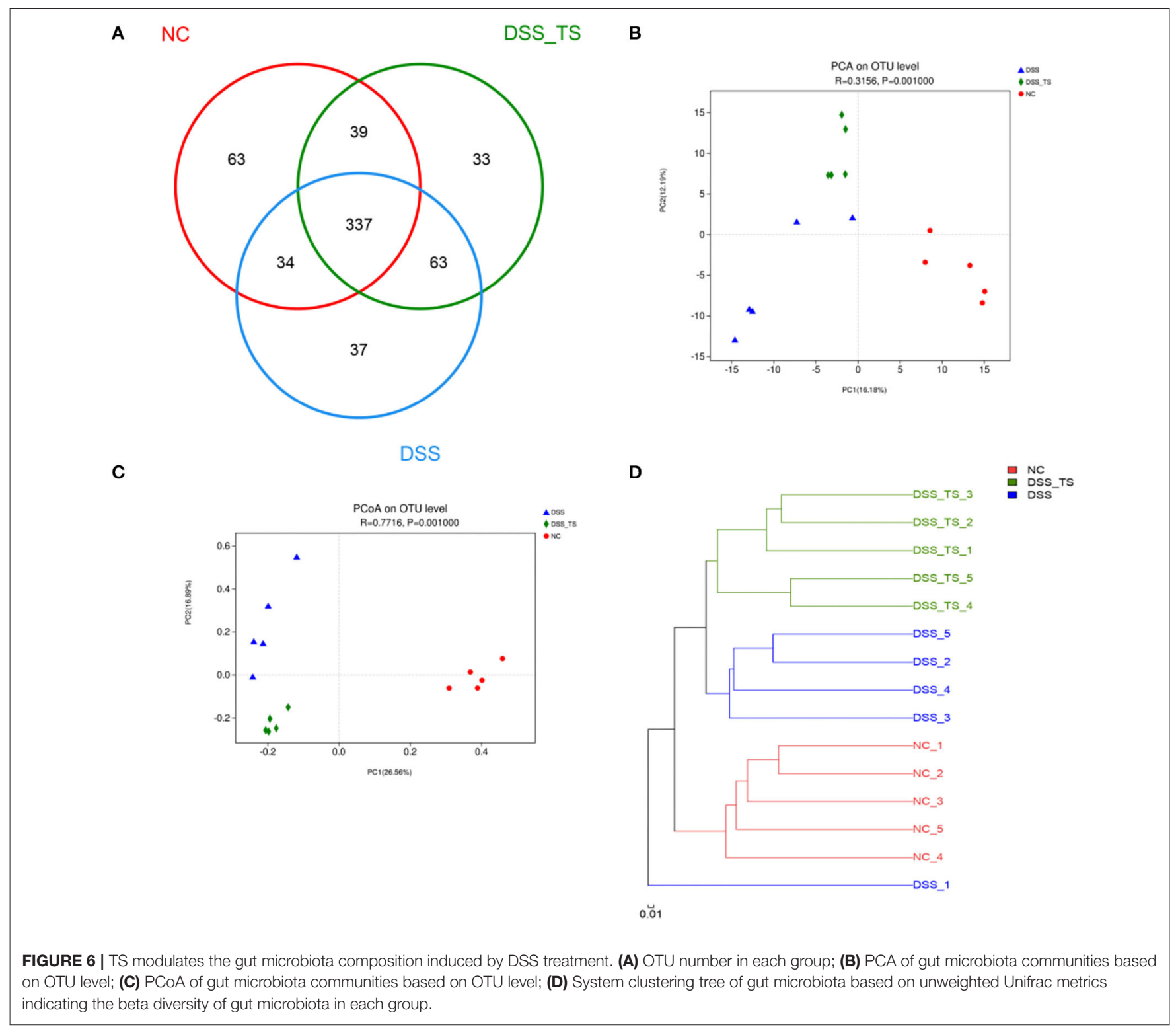

TS exerted an anti-inflammatory effect on DSS-induced colitis and might have potential to prevent and cure IBD patients.

Induced activation of p65 in response to a variety of stimuli is typically transient but sufficient to upregulate transactivation of target genes of diverse activities such as the cellular proliferation, inflammatory cytokines $(15,16)$. Consequently, TS inactivated NF- $\kappa$ B pathway signaling, leading to the decline of inflammatory cytokines and finally promoting the intestinal physiology and pathophysiology. The production of TNF- $\alpha$, IL- $1 \beta$, and IL6 plays leading roles in the initiation and progression of colitis, and some of them have been regarded as a logical target for IBD therapy $(8,17)$. Thus, we hypothesized that TS attenuated the inflammatory response in the gut by inhibiting NF- $\kappa \mathrm{B}$ activation.

IL-6 is released upon stimulation of inflammatory cells to activate signaling pathways such as STAT3 (18). STAT3 is activated in both the epithelial and hematopoietic compartments in $\operatorname{IBD}(19,20)$. Consistently, our results showed that STAT3 was more activated in the DSS group compared with DSS+TS group. The role of STAT3 in the epithelial compartment is complex, it is important for inducing return to homeostasis following acute infection, while at the same time it involves the induction of cytokines and chemokines expression. Perhaps, TS suppressed inflammatory cytokines partly via inactivating STAT3 in DSS-induced colitis mice.

Colitis is an immune-related disease caused by neutrophil and macrophage infiltration in the colonic lamina propria $(21,22)$, but the effect of TS on immune cells is unclear. Our study found that dietary TS ameliorated neutrophil accumulation in the colon of DSS-induced colitis mice which was accompanied with decreased TNF- $\alpha$, IL- 6 , and IL- $1 \beta$ expression. The microbiota of IBD patients can change the intestinal CD4+T 


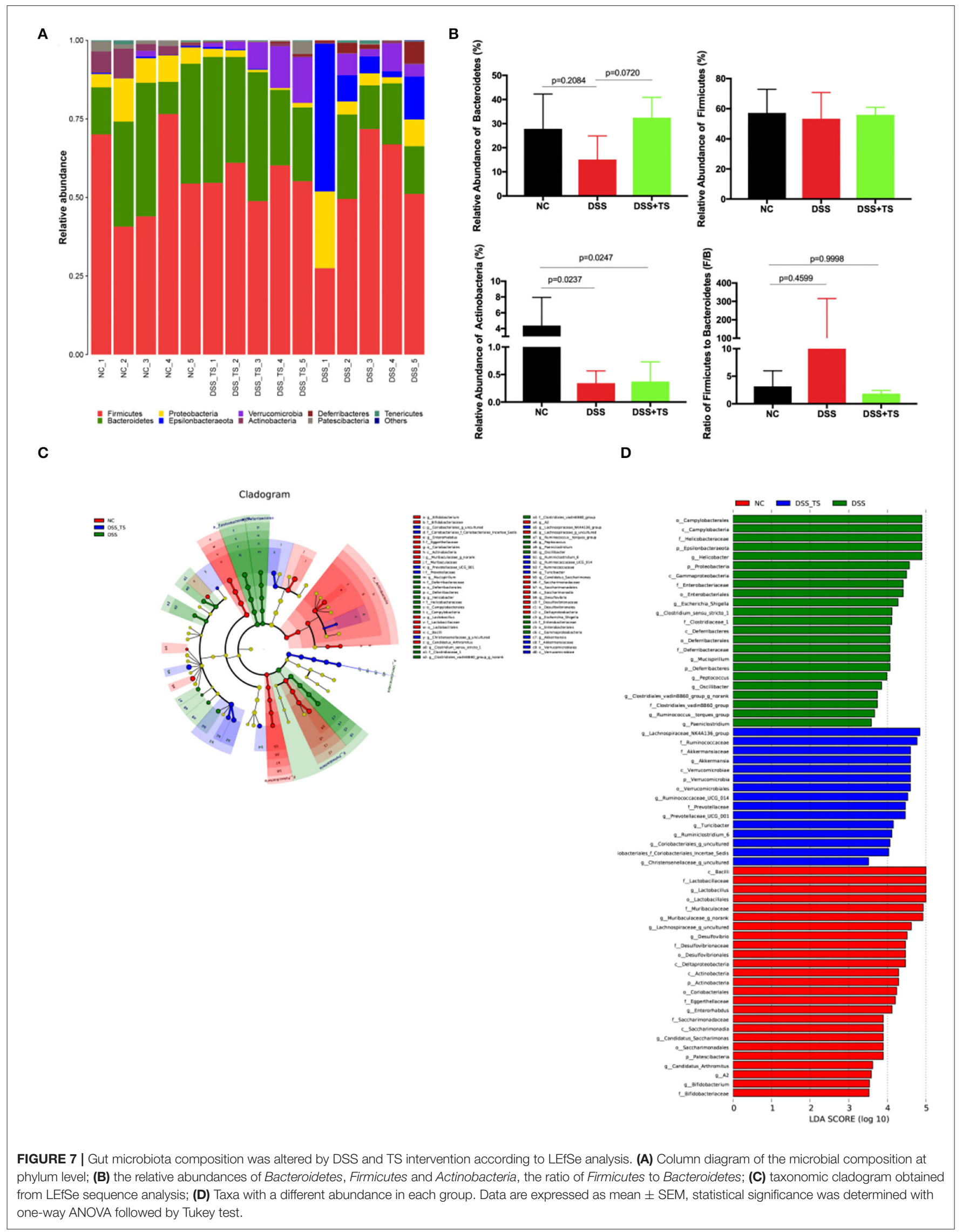


A

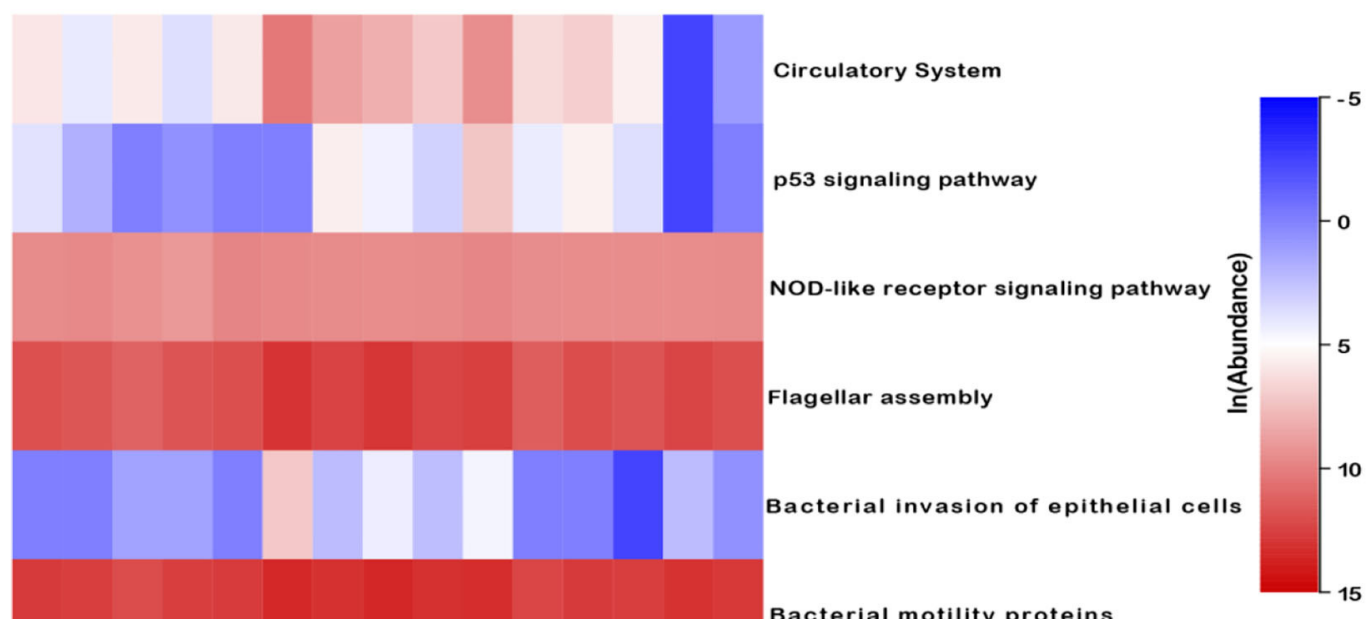

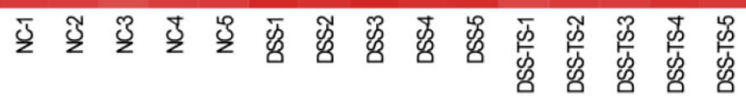

B

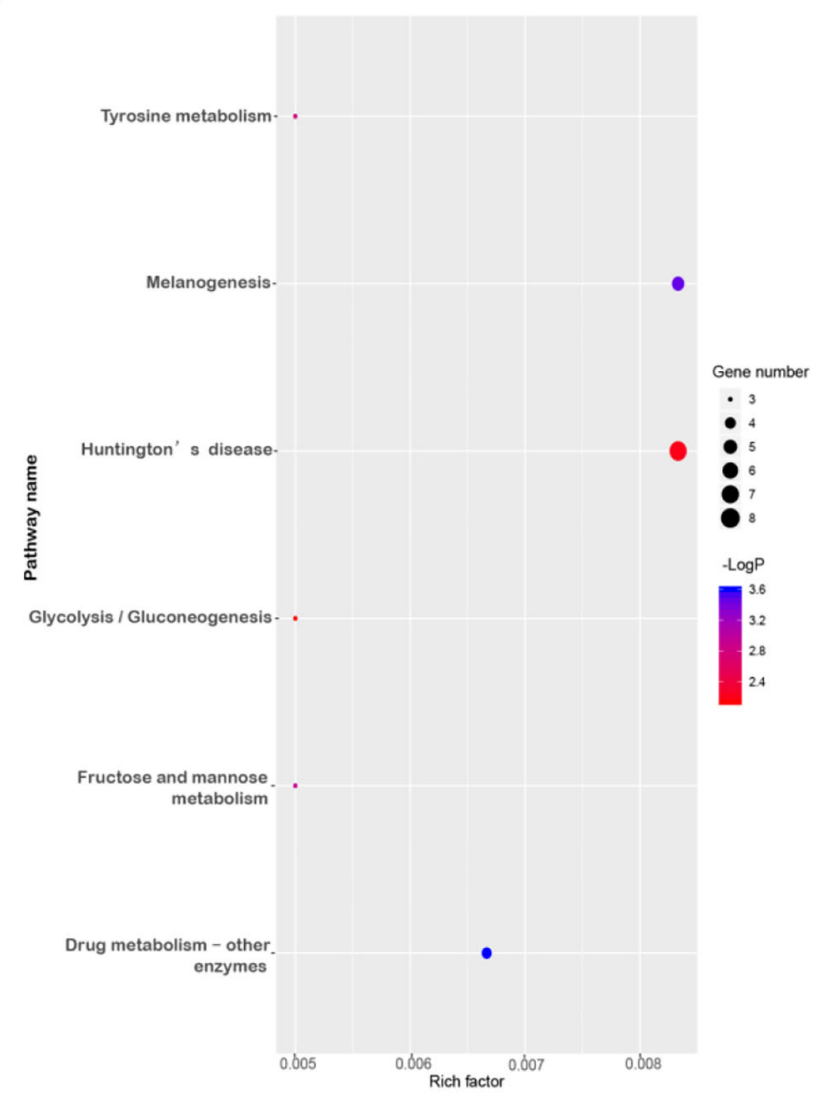

C

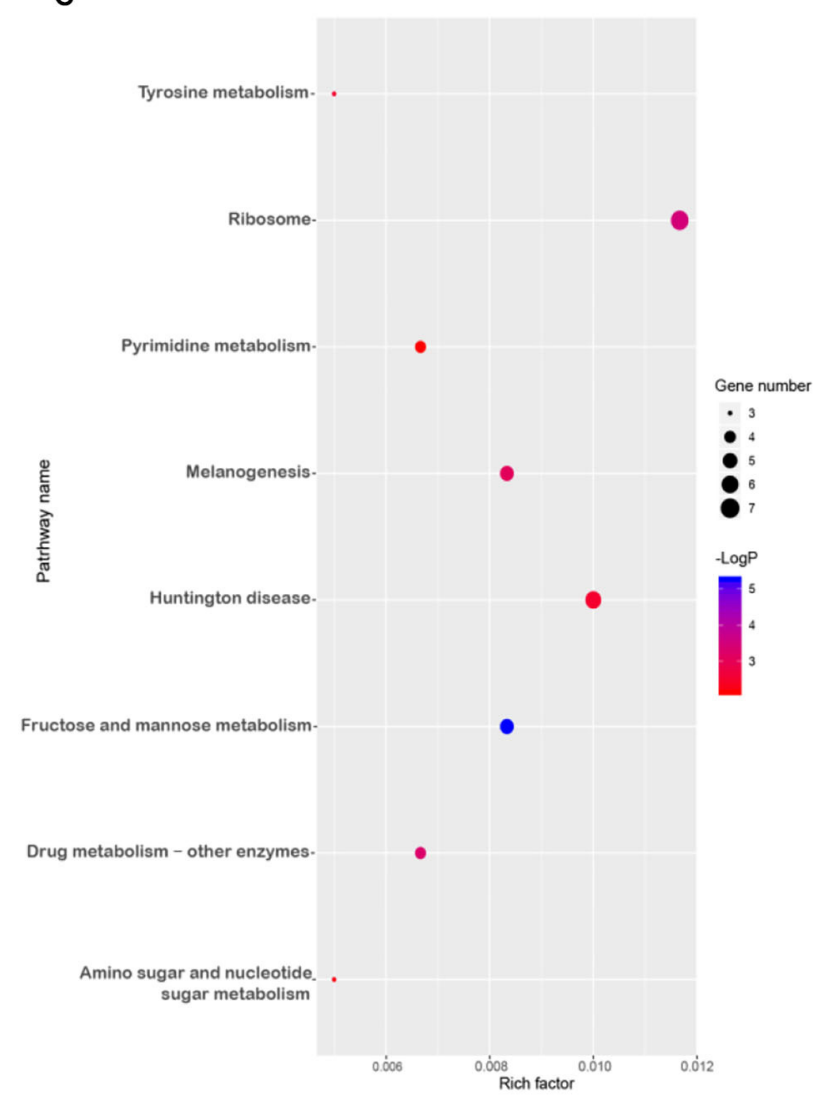

FIGURE 8 | The effect of TS on the microbial community function predicted by PICRUSt. (A) Heatmap shows the relative abundance of pathways in different groups; Bubble chart shows the gene abundances in pathways (B) DSS group relative to NC group, (C) DSS+TS group relative to DSS group.

cell homeostasis of the sterile recipient mice (23). Our results also demonstrated that colonization with $\mathrm{CD} 4+\mathrm{T}$ cells was dramatically increased in DSS-induced colitis, and TS inhibited the recruitment of $\mathrm{CD} 4+\mathrm{T}$ cells. In conclusion, TS mainly regulates neutrophil infiltration to protect mice against DSSinduced colitis. 
A

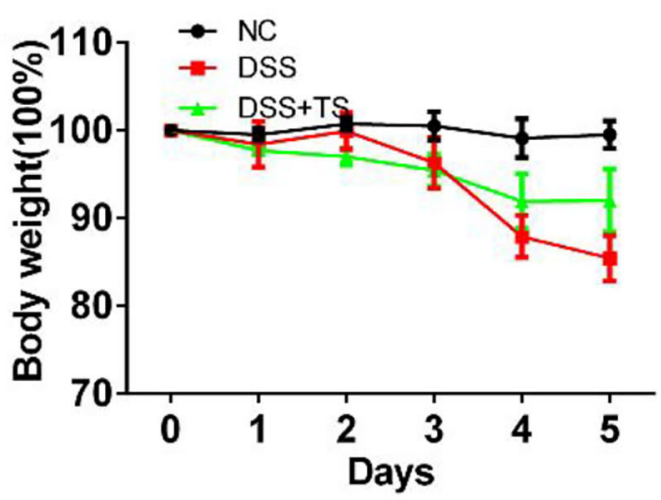

C

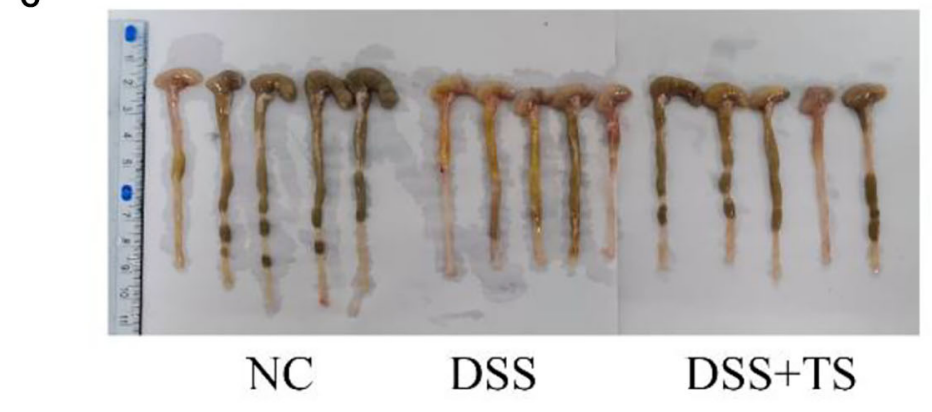

E

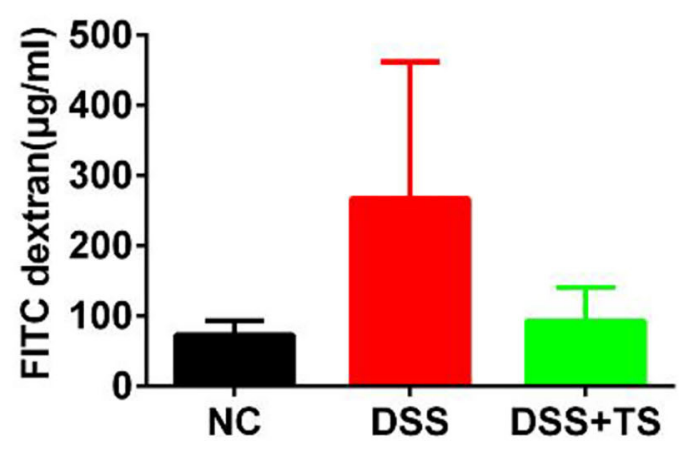

B

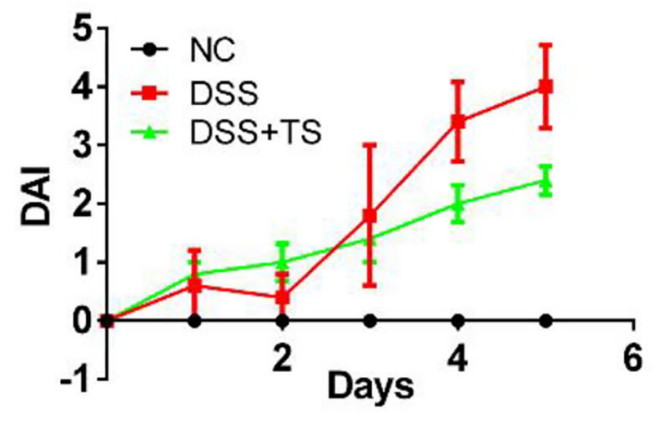

D

FIGURE 9 | The effect of microbiota induced by TS in DSS-induced colitis in mice. (A) Body weight; (B) DAI score; (C) Images of colon; (D) colon length; (E) detection of FITC-dextran in serum. Data are expressed as the mean \pm SEM $(n=5)$, statistical significance was determined with one-way ANOVA followed by Tukey test, and $p$-values are as follows: ${ }^{\star} p<0.05$.

Besides immune system disorders, one typical symptom of IBD is weakening of the mucus barrier, leading to further gut bacterial disruption and inappropriate immune activation (24). The colonic epithelium contains several differentiated cell types like enterocytes and goblet cells, each of which responsible for absorption, mucus production and antigen sampling $(25,26)$. Defects in mucus production and tight junction have been described in IBD $(7,27,28)$. DSSinduced colitis was also found to be associated with the decreased expression of Muc2, Claudin-1, and ZO-1. TS significantly ameliorated these markers, which could preserve the mucus barrier away from DSS invasion via decreasing proinflammatory cytokines levels. Relevant studies reported the possible mechanisms and even considered that the intestinal integrity might be a crucial target for the treatment of IBD (29).

Gut microbiota is increasingly thought of as a key factor in the cause and therapy target of IBD. The immune system can be triggered by gut microbiota, showing a cause-and-effect relationship with the disease (30). IBD has been shown to be 
A

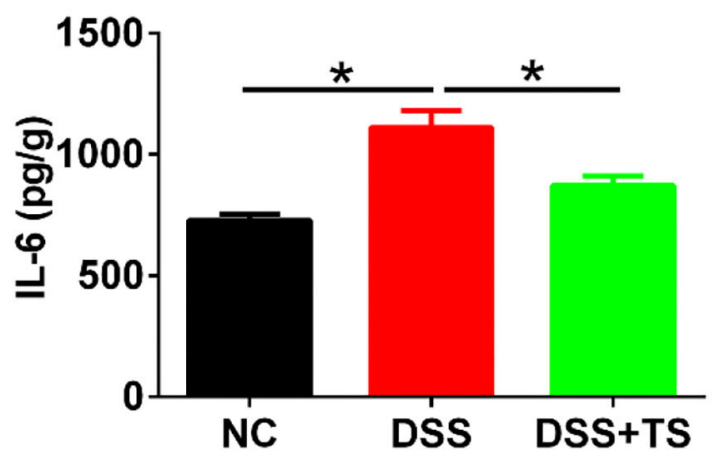

B

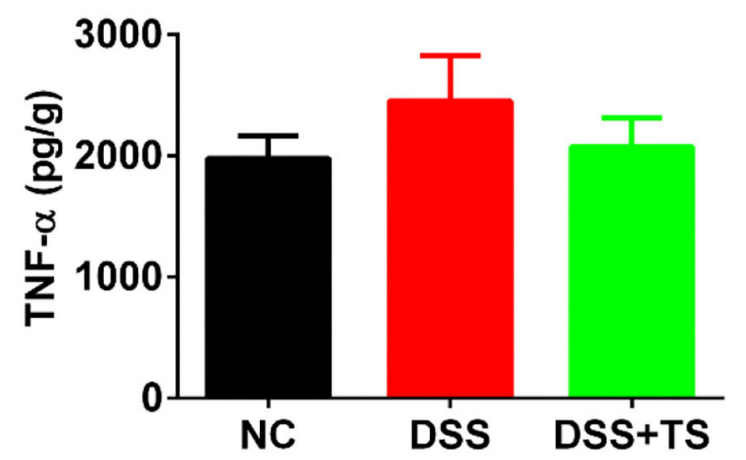

C

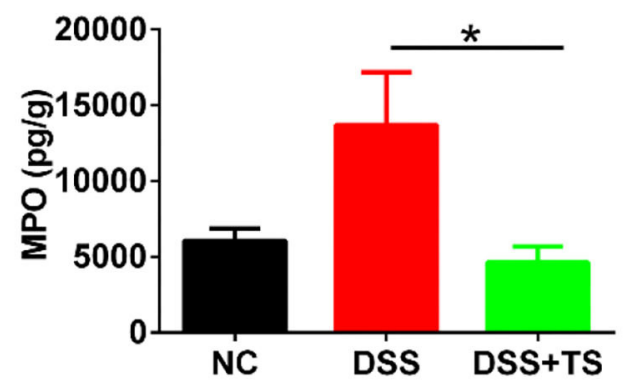

FIGURE 10 | The association of microbiota induced by TS with gut inflammation. (A,B) the protein levels of IL-6 and TNF- $\alpha$ by ELISA; (C) the protein levels of MPO by ELISA. Data are expressed as mean $\pm \operatorname{SEM}(n=5)$, statistical significance was determined with one-way ANOVA followed by Tukey test, and $p$-values are as follows: ${ }^{*} p<0.05$.

associated with change in the composition and metabolism of the gut microbiota due to a shift between commensal and pathogenic microorganisms $(31,32)$. Although there is no conclusive direct causal relationship between IBD and dysbiosis, some specific bacteria have abilities to induce pro-inflammatory or protective effects and the chronic inflammation has access to shape the gut microbiota (33-35). The underlying mechanisms still need further study. In this study, we found that the structure and composition of the gut microbiota underwent changes in mice with DSS-induced colitis. At the phylum level, the F/B ratio was increased, which is consistent with the finding of other studies $(7,36,37)$. TS could reverse the $\mathrm{F} / \mathrm{B}$ ratio to correct the disorder of the intestinal microbiota induced by DSS. As inflammation is an oxidative state, it might be expected to promote the outgrowth of aerotolerant taxa such as Actinobacteria (38). A cohort study reported a significant increase in Actinobacteria in UC patients (39). Whereas, it has been reported previously in IBD that there is a decrease in Actinobacteria abundance compared to healthy subjects (40). Therefore, previous reports on the abundance of actinomycetes were not consistent in IBD patients. Since in our analysis the relative abundance of Actinobacteria was also significantly increased in DSS-induced colitis, this aspect should be further investigated.
Additionally, the DSS group showed higher abundance of pathogenic microbiota (e.g., Campylobacteria, Proteobacteria, Helicobacter, and Enterobacteriaceae) and lower beneficial microbiota (e.g., Lactobacillus and Bifidobacterium) compared with the NC group in our study. Campylobacteria has emerged as a putative player in the pathogenesis of IBD due to its extraordinary and diverse pathogenic capabilities (41). Proteobacteria is considered as a typical pathogen associated with IBD pathogenesis (37). Lupp et al. reported that colonic inflammation supported the growth of some specific aerobic bacteria in the gut, especially the Enterobacteriaceae family (42). In our research, the Enterobacteriaceae family was abundant and even Escherichia-Shigella became the dominant bacteria in the DSS group. Shigella is considered the crucial factor leading to the increased intestinal permeability and even worsening the disease $(43,44)$ Akkermansia muciniphila is known as a member of Akkermansia with the ability to maintain the intestinal integrity and exhibit anti-inflammatory responses $(45,46)$.

Meanwhile, significantly different functional profiles between different groups were predicted by PICRUSt to highlight the importance of the gut microbiota in these metabolic pathways. Our results showed relatively high abundances in flagellar assembly, bacterial invasion of epithelial cells and 
bacterial motility proteins in the DSS group, which could be significantly reversed by TS treatment. These pathways play important roles in bacteria adhesion and motility. Adhesion to mucus and invasion of epithelial cells are essential for infection by pathogenic bacteria. Studies have shown that infection with pathogenic bacteria such as Salmonella and $C$. difficile promotes the onset of $\operatorname{IBD}(47,48)$. In TS treatment colitis, a decrease in the identification of predictive microbial signals could also allow for refinement of TS that may deliver specific anti-inflammatory role that may be of benefit in ameliorating inflammation. Therefore, the inhibitory effect of TS on bacterial adhesion and invasion to the host may have a therapeutic value in IBD. The predominantly glycolytic form of metabolism shared by Polymorphonuclear leukocytes (PMNs) is thought to ensure their survival and function in the hypoxic, often anoxic environment of deep inflammatory foci. In DSS-induced colitis, the increase of glycolytic may reflect the recruitment of PMNs. It was found in the present study that the intervention of TS significantly improved this function. In addition, compared with the DSS group, TS treatment led to changes in more complex functional pathways, such as pyrimidine metabolism, amino sugar and nucleotide sugar metabolism and ribosome. These results demonstrated the ability of TS from tea to adjust metabolic pathways of the gut microbiota in IBD, though further experiments are necessary to confirm identification of these pathways and related genes.

In conclusion, our study demonstrated that DSS treatment disturbed the gut microbiota by triggering an immune response, and TS treatment significantly attenuated the severity of inflammation, modulated the metabolism pathways and restored the unbalanced microbiota composition to a normal condition in mice with DSS-induced colitis. In addition, we delineated the features of the intestinal microbiota that are associated with IBD. Although our study demonstrated an association between TS treatment and change of the microbiota, causative studies are needed to completely understand the role of the gut microbiota during TS treatment. Furthermore, our study provided the better understand the role of the gut microbiota of TS treatment

\section{REFERENCES}

1. Ng SC. Epidemiology of inflammatory bowel disease: focus on Asia. Best Pract Res Clin Gastroenterol. (2014) 28:363-72. doi: 10.1016/j.bpg.2014.04.003

2. Ng SC, Shi HY, Hamidi N, Underwood FE, Tang W, Benchimol EI, et al. Worldwide incidence and prevalence of inflammatory bowel disease in the 21st century: a systematic review of population-based studies. Lancet. (2017) 390:2769-78. doi: 10.1016/S0140-6736(17)32448-0

3. Desai D, Shah S, Deshmukh A, Abraham P, Joshi A, Gupta T, et al. Colorectal cancers in ulcerative colitis from a low-prevalence area for colon cancer. World J Gastroenterol. (2015) 21:3644-9. doi: 10.3748/wjg.v21.i12. 3644

4. Franzosa EA, Sirota-Madi A, Avila-Pacheco J, Fornelos N, Haiser $\mathrm{HJ}$, Reinker $\mathrm{S}$, et al. Gut microbiome structure and metabolic activity in inflammatory bowel disease. Nat Microbiol. (2019) 4:293-305. doi: 10.1038/s41564-018-0306-4

5. Imhann F, Vich Vila A, Bonder MJ, Fu J, Gevers D, Visschedijk MC, et al. Interplay of host genetics and gut microbiota underlying the onset and in controlling the induction of immune system in mice with DSS-induced colitis. Our data also provide a foundation for future study at the transcriptional level to better establish the metabolomics profiles associated with IBD. This study adds to our understanding of interactions between the immune system, the microbiota and the host, and provides the basis for future clinical study of microbiota manipulation and the development of new therapeutic strategies for IBD.

\section{DATA AVAILABILITY STATEMENT}

The original contributions presented in the study are included in the article/supplementary materials, further inquiries can be directed to the corresponding author/s.

\section{ETHICS STATEMENT}

The animal study was reviewed and approved by Laboratory Animal Ethics Committee of Xuzhou Medical University (201908A005).

\section{AUTHOR CONTRIBUTIONS}

$\mathrm{HY}$ is responsible for providing the overall idea. RC drafted the paper. $\mathrm{ZK}$ and $\mathrm{SQ}$ are in charge of experimental design. $\mathrm{YC}$ is responsible for data collation. $\mathrm{CC}$ is in charge of animal experiments. BG made important changes to the paper. All authors contributed to the article and approved the submitted version.

\section{FUNDING}

This research was supported by the National Natural Science Foundation of China (81871734 and 81471994), Jiangsu Provincial Natural Science Foundation (BK20151154), Jiangsu Provincial Medical Talent (ZDRCA2016053), the Xuzhou Science and Technology planning Project (KC20116), and Xuzhou Medical University Excellent Talent Introduction Project (D2019030). clinical presentation of inflammatory bowel disease. Gut. (2018) 67:10819. doi: 10.1136/gutjnl-2016-312135

6. Moschen AR, Tilg H, Raine T. IL-12, IL-23 and IL-17 in IBD: immunobiology and therapeutic targeting. Nat Rev Gastroenterol Hepatol. (2019) 16:18596. doi: 10.1038/s41575-018-0084-8

7. Peng Y, Yan Y, Wan P, Chen D, Ding Y, Ran L, et al. Gut microbiota modulation and anti-inflammatory properties of anthocyanins from the fruits of Lycium ruthenicum murray in dextran sodium sulfate-induced colitis in mice. Free Radic Biol Med. (2019) 136:96-108. doi: 10.1016/j.freeradbiomed.2019. 04.005

8. Mowat C, Cole A, Windsor A, Ahmad T, Arnott I, Driscoll R, et al. Guidelines for the management of inflammatory bowel disease in adults. Gut. (2011) 60:571-607. doi: 10.1136/gut.2010. 224154

9. Salminen A, Lehtonen $\mathrm{M}$, Suuronen $\mathrm{T}$, Kaarniranta K, Huuskonen J. Terpenoids: natural inhibitors of NF- $\mathrm{B}$ signaling with antiinflammatory and anticancer potential. Cell Mol Life Sci. (2008) 65:2979-99. doi: 10.1007/s00018-008-8103-5 
10. Wang S, Huang XF, Zhang P, Newell KA, Wang H, Zheng K, et al. Dietary teasaponin ameliorates alteration of gut microbiota and cognitive decline in diet-induced obese mice. Sci Rep. (2017) 7:12203. doi: 10.1038/s41598-017-12156-2

11. Yu Y, Wu Y, Szabo A, Wu Z, Wang H, Li D, et al. Teasaponin reduces inflammation and central leptin resistance in diet-induced obese male mice. Endocrinology. (2013) 154:3130-40. doi: 10.1210/en.20131218

12. Viennois E, Chen F, Laroui H, Baker MT, Merlin D. Dextran sodium sulfate inhibits the activities of both polymerase and reverse transcriptase: lithium chloride purification, a rapid and efficient technique to purify RNA. BMC Res Notes. (2013) 6:360. doi: 10.1186/1756-0500-6360

13. Stillie R, Stadnyk AW. Role of TNF receptors, TNFR1 and TNFR2, in dextran sodium sulfate-induced colitis. Inflammat Bowel Dis. (2009) 15:151525. doi: 10.1002/ibd.20951

14. Park YH, Kim N, Shim YK, Choi YJ, Nam RH, Choi YJ, et al. Adequate dextran sodium sulfate-induced colitis model in mice and effective outcome measurement method. J Cancer Prev. (2015) 20:2607. doi: 10.15430/JCP.2015.20.4.260

15. Basak S, Behar M, Hoffmann A. Lessons from mathematically modeling the NF-кB pathway. Immunol Rev. (2012) 246:22138. doi: 10.1111/j.1600-065X.2011.01092.x

16. Yde P, Mengel B, Jensen MH, Krishna S, Trusina A. Modeling the NF-kappaB mediated inflammatory response predicts cytokine waves in tissue. BMC Syst Biol. (2011) 5:115. doi: 10.1186/1752-0509-5-115

17. Neurath MF. Current and emerging therapeutic targets for IBD. Nat Rev Gastroenterol Hepatol. (2017) 14:269-78. doi: 10.1038/nrgastro. 2016.208

18. Sommer J, Engelowski E, Baran P, Garbers C, Floss DM, Scheller J. Interleukin-6, but not the interleukin-6 receptor plays a role in recovery from dextran sodium sulfate-induced colitis. Int J Mol Med. (2014) 34:65160. doi: 10.3892/ijmm.2014.1825

19. Mitsuyama K, Matsumoto S, Rose-John S, Suzuki A, Hara T, Tomiyasu $\mathrm{N}$, et al. STAT3 activation via interleukin 6 trans-signalling contributes to ileitis in SAMP1/Yit mice. Gut. (2006) 55:1263-9. doi: 10.1136/gut.2005. 079343

20. Mudter J, Weigmann B, Bartsch B, Kiesslich R, Strand D, Galle PR, et al. Activation pattern of signal transducers and activators of transcription (STAT) factors in inflammatory bowel diseases. Am J Gastroenterol. (2005) 100:6472. doi: 10.1111/j.1572-0241.2005.40615.x

21. Bousquet MS, Ratnayake R, Pope JL, Chen QY, Zhu F, Chen S, et al. Seaweed natural products modify the host inflammatory response via Nrf2 signaling and alter colon microbiota composition and gene expression. Free Radic Biol Med. (2020) 146:306-23. doi: 10.1016/j.freeradbiomed.2019. 09.013

22. Gao X, Cao Q, Cheng Y, Zhao D, Wang Z, Yang H, et al. Chronic stress promotes colitis by disturbing the gut microbiota and triggering immune system response. Proc Natl Acad Sci USA. (2018) 115:E29609. doi: 10.1073/pnas.1720696115

23. Britton GJ, Contijoch EJ, Mogno I, Vennaro OH, Llewellyn SR, Ng R, et al. Microbiotas from humans with inflammatory bowel disease alter the balance of gut Th17 and ROR $\gamma \mathrm{t}+$ regulatory $\mathrm{T}$ cells and exacerbate colitis in mice. Immunity. (2019) 50:212-24.e4. doi: 10.1016/j.immuni.2018. 12.015

24. Ramos GP, Papadakis KA. Mechanisms of disease: inflammatory bowel diseases. Mayo Clin Proc. (2019) 94:15565. doi: 10.1016/j.mayocp.2018.09.013

25. Gerbe F, Legraverend C, Jay P. The intestinal epithelium tuft cells: specification and function. Cell Mol Life Sci. (2012) 69:2907-17. doi: 10.1007/s00018-012-0984-7

26. Martens EC, Neumann M, Desai MS. Interactions of commensal and pathogenic microorganisms with the intestinal mucosal barrier. Nat Rev Microbiol. (2018) 16:457-70. doi: 10.1038/s41579-018-0036-x

27. Capaldo CT, Powell DN, Kalman D. Layered defense: how mucus and tight junctions seal the intestinal barrier. J Mol Med. (2017) 95:92734. doi: 10.1007/s00109-017-1557-x
28. McGuckin MA, Eri R, Simms LA, Florin TH, RadfordSmith G. Intestinal barrier dysfunction in inflammatory bowel diseases. Inflamm Bowel Dis. (2009) 15:100-13. doi: 10.1002/ibd. 20539

29. Woo JK, Choi S, Kang JH, Kim DE, Hurh BS, Jeon JE, et al. Fermented barley and soybean (BS) mixture enhances intestinal barrier function in dextran sulfate sodium (DSS)-induced colitis mouse model. BMC Complement Altern Med. (2016) 16:498. doi: 10.1186/s12906-0161479-0

30. Cekanaviciute E, Yoo BB, Runia TF, Debelius JW, Singh S, Nelson CA, et al. Gut bacteria from multiple sclerosis patients modulate human $\mathrm{T}$ cells and exacerbate symptoms in mouse models. Proc Natl Acad Sci USA. (2017) 114:10713-8. doi: 10.1073/pnas.17112 35114

31. Mosca A, Leclerc M, Hugot JP. Gut microbiota diversity and human diseases: should we reintroduce key predators in our ecosystem? Front Microbiol. (2016) 7:455. doi: 10.3389/fmicb.2016.00455

32. Manichanh C, Borruel N, Casellas F, Guarner F. The gut microbiota in IBD. Nat Rev Gastroenterol Hepatol. (2012) 9:599-608. doi: 10.1038/nrgastro.2012.152

33. Zhang SL, Wang SN, Miao CY. Influence of microbiota on intestinal immune system in ulcerative colitis and its intervention. Front Immunol. (2017) 8:1674. doi: 10.3389/fimmu.2017.01674

34. Prosberg M, Bendtsen F, Vind I, Petersen AM, Gluud LL. The association between the gut microbiota and the inflammatory bowel disease activity: a systematic review and meta-analysis. Scand J Gastroenterol. (2016) 51:140715. doi: 10.1080/00365521.2016.1216587

35. Goodman AL, Jeon SG, Kayama H, Takahashi T, Asahara T, Tsuji H, et al. Probiotic Bifidobacterium breve induces IL-10-producing Tr1 cells in the colon. PLoS Pathog. (2012) 8:e1002714. doi: 10.1371/journal.ppat.10 02714

36. Nishida A, Inoue R, Inatomi O, Bamba S, Naito Y, Andoh A. Gut microbiota in the pathogenesis of inflammatory bowel disease. Clin J Gastroenterol. (2018) 11:1-10. doi: 10.1007/s12328-017-0813-5

37. Zhang XJ, Yuan ZW, Qu C, Yu XT, Huang T, Chen PV, et al. Palmatine ameliorated murine colitis by suppressing tryptophan metabolism and regulating gut microbiota. Pharmacol Res. (2018) 137:34-46. doi: 10.1016/j.phrs.2018.09.010

38. Ni J, Wu GD, Albenberg L, Tomov VT. Gut microbiota and IBD: causation or correlation? Gastroenterol Hepatol. (2017) 14:573-84. doi: 10.1038/nrgastro.2017.88

39. Santoru ML, Piras C, Murgia A, Palmas V, Camboni T, Liggi S, et al. Cross sectional evaluation of the gut-microbiome metabolome axis in an Italian cohort of IBD patients. Sci Rep. (2017) 7:9523. doi: 10.1038/s41598-017-10034-5

40. Marteau P. Bacterial flora in inflammatory bowel disease. Dig Dis. (2009) 27:99-103. doi: 10.1159/000268128

41. Kaakoush NO, Mitchell HM, Man SM. Role of emerging Campylobacter species in inflammatory bowel diseases. Inflamm Bowel Dis. (2014) 20:218997. doi: 10.1097/MIB.0000000000000074

42. Lupp C, Robertson ML, Wickham ME, Sekirov I, Champion OL, Gaynor EC, et al. Host-mediated inflammation disrupts the intestinal microbiota and promotes the overgrowth of Enterobacteriaceae. Cell Host Microbe. (2007) 2:119-29. doi: 10.1016/j.chom.2007.06.010

43. Kang S, Denman SE, Morrison M, Yu Z, Dore J, Leclerc M, et al. Dysbiosis of fecal microbiota in Crohn's disease patients as revealed by a custom phylogenetic microarray. Inflamm Bowel Dis. (2010) 16:203442. doi: 10.1002/ibd.21319

44. Bian X, Wu W, Yang L, Lv L, Wang Q, Li Y, et al. Administration of Akkermansia muciniphila ameliorates dextran sulfate sodiuminduced ulcerative colitis in mice. Front Microbiol. (2019) 10:2259. doi: 10.3389/fmicb.2019.02259

45. Derrien M, Belzer C, de Vos WM. Akkermansia muciniphila and its role in regulating host functions. Microbial Pathog. (2017) 106:1718. doi: 10.1016/j.micpath.2016.02.005

46. Geerlings SY, Kostopoulos I, de Vos WM, Belzer C. Akkermansia muciniphila in the human gastrointestinal tract: when, where, and 
how? Microorganisms. (2018) 6:75. doi: 10.3390/microorganisms 6030075

47. Zhang T, Lin QY, Fei JX, Zhang Y, Lin MY, Jiang SH, et al. Clostridium difficile infection worsen outcome of hospitalized patients with inflammatory bowel disease. Sci Rep. (2016) 6:29791. doi: 10.1038/srep 29791

48. Schultz BM, Paduro CA, Salazar GA, Salazar-Echegarai FJ, Sebastián VP, Riedel CA, et al. A potential role of salmonella infection in the onset of inflammatory bowel diseases. Front Immunol. (2017) 8:191. doi: 10.3389/fimmu.2017.
Conflict of Interest: The authors declare that the research was conducted in the absence of any commercial or financial relationships that could be construed as a potential conflict of interest.

Copyright (C) 2020 Yang, Cai, Kong, Chen, Cheng, Qi and Gu. This is an open-access article distributed under the terms of the Creative Commons Attribution License (CC $B Y)$. The use, distribution or reproduction in other forums is permitted, provided the original author(s) and the copyright owner(s) are credited and that the original publication in this journal is cited, in accordance with accepted academic practice. No use, distribution or reproduction is permitted which does not comply with these terms. 